\title{
Divergence-Free MHD on Unstructured Meshes Using High Order Finite Volume Schemes Based on Multidimensional Riemann Solvers
}

\author{
Dinshaw S. Balsara ${ }^{1}$ and Michael Dumbser ${ }^{2}$ \\ ${ }^{1}$ Physics Department, University of Notre Dame, USA (ㅁalsara@nd.edu) \\ ${ }^{2}$ Laboratory of Applied Mathematics, Department of Civil, Environmental and \\ Mechanical Engineering, University of Trento, Italy (michael.dumbser@unitn.it)
}

\begin{abstract}
Several advances have been reported in the recent literature on divergence-free finite volume schemes for Magnetohydrodynamics (MHD). Almost all of these advances are restricted to structured meshes. To retain full geometric versatility, however, it is also very important to make analogous advances in divergence-free schemes for MHD on unstructured meshes. Such schemes utilize a staggered Yee-type mesh, where all hydrodynamic quantities (mass, momentum and energy density) are cell-centered, while the magnetic fields are face-centered and the electric fields, which are so useful for the time update of the magnetic field, are centered at the edges.

Three important advances are brought together in this paper in order to make it possible to have high order accurate finite volume schemes for the MHD equations on unstructured meshes. First, it is shown that a divergence-free WENO reconstruction of the magnetic field can be developed for unstructured meshes in two and three space dimensions using a classical cellcentered WENO algorithm, without the need to do a WENO reconstruction for the magnetic field on the faces. This is achieved via a novel constrained $\mathrm{L}_{2}$-projection operator that is used in each time step as a postprocessor of the cell-centered WENO reconstruction so that the magnetic field becomes locally and globally divergence free. Second, it is shown that recently-developed genuinely multidimensional Riemann solvers (called MuSIC Riemann solvers) can be used on unstructured meshes to obtain a multidimensionally upwinded representation of the electric field at each edge. Third, the above two innovations work well together with a high order accurate one-step ADER time stepping strategy, which requires the divergence-free nonlinear WENO reconstruction procedure to be carried out only once per time step.

The resulting divergence-free ADER-WENO schemes with MuSIC Riemann solvers give us an efficient and easily-implemented strategy for divergence-free MHD on unstructured meshes. Several stringent two- and three-dimensional problems are shown to work well with the methods presented here.
\end{abstract}




\section{I) Introduction}

The magnetohydrodynamic (MHD) equations have become increasingly important in astrophysics, space physics and plasma physics. MHD is also the simplest approximation in a hierarchy of approximations for modelling ionized plasmas. Many novel computational insights, which are inapplicable to computational fluid dynamics, have to be developed for the numerical solution of the compressible MHD equations. Those insights can also be used in other more sophisticated approximations for modelling ionized plasmas. For MHD, the ionized plasma couples to the magnetic field which evolves according to Faraday's law:

$$
\frac{\partial \mathbf{B}}{\partial t}+\nabla \times \mathbf{E}=0
$$

In the ideal MHD approximation, we have $\mathbf{E}=-\mathbf{v} \times \mathbf{B}$, where $\mathbf{B}$ is the magnetic field, $\mathbf{E}$ is the electric field and $\mathbf{v}$ is the fluid velocity. Since the magnetic field must be divergence-free at the initial time, i.e. $\nabla \square \mathbf{B}=0$, Faraday's law ensures that the magnetic field remains divergence-free for all time. Physically, this means that no magnetic monopoles can exist. Good numerical methods for MHD ought to retain this property. In a very influential paper, Brackbill \& Barnes [22] showed that if a non-zero divergence of $\mathbf{B}$ is allowed to build up in the computational domain, it can lead to unphysical plasma transport parallel to the magnetic field.

To promote fidelity with the physics, several schemes have been devised that keep the magnetic field divergence-free. Fundamentally, all these methods use a staggered Yee-type [74] mesh where the normal components of the magnetic fields reside in the faces of the mesh and the electric fields reside at the edges of the mesh. Such schemes are also known as constrained transport schemes because they transport the magnetic field consistent with the divergence-free constraint. Early constrained transport schemes were devised without the use of higher order Godunov methods (Brecht et al. [23], DeVore [33], Evans\& Hawley [41]).

Over the last decade or two, several effective one-dimensional Riemann solvers have become available for numerical MHD (Brio \& Wu [24], Zachary et al. [75], Dai \& Woodward [30], Ryu \& Jones [62], Roe \& Balsara [61], Cargo \& Gallice [25], Balsara [3], Falle et al. [42], Gurski [45], Li [53], Miyoshi \& Kusano [56]). This has resulted in several Godunov schemes for numerical MHD. Many of the earlier such schemes did not respect the divergence-free constraint (Brackbill\& Barnes [22], Zachary et al. [75], Crockett et al. [29], Balsara [4], Balbas et al. [2]). Most such schemes resort to some strategy for reducing the unbounded growth of divergence in the magnetic field. Hodge projection approaches have been suggested (Zachary et al. [75], Balsara [4]) and the deficiencies of the Hodge projection have been catalogued by Balsara \& Kim [10]. The Powell [59] source term formulation advects away any divergence that might build up, but it only does so by introducing source terms that destroy the conservation properties of the momentum and energy equations. The generalized Lagrange multiplier (GLM) approach by Dedneret al. [32] does provide conservation of momentum and energy, however Mocz et al. 
[57] present some problems where the method proves deficient. The GLM method has the further problem that the speed with which the GLM field needs to propagate has to be faster than the fastest speed in the problem. In some simulations, this top speed can grow by orders of magnitude as the simulation evolves, making it difficult to predict the top speed. Modern higher order Godunov schemes for numerical MHD tend to incorporate the divergence-free property for the magnetic field (Dai \& Woodward [31], Ryu et al. [63], Balsara \& Spicer [6], Toth [69], Londrillo \& DelZanna [54], Gardiner \& Stone [43], [44], Balsara [7], [8], [9], Lee [50]). Balsara\& Spicer [6] suggested that the dualism between the components of the numerical flux and the electric field can be used to obtain the electric field at the edges of each zone. All higher order, divergence-free Godunov schemes for numerical MHD have incorporated this plan in one form or another.

A reading of the later sections of Balsara \& Spicer [6] shows that the electric field has to be obtained in a multidimensional manner. Let us focus on the equations of MHD in flux form to understand the issue.

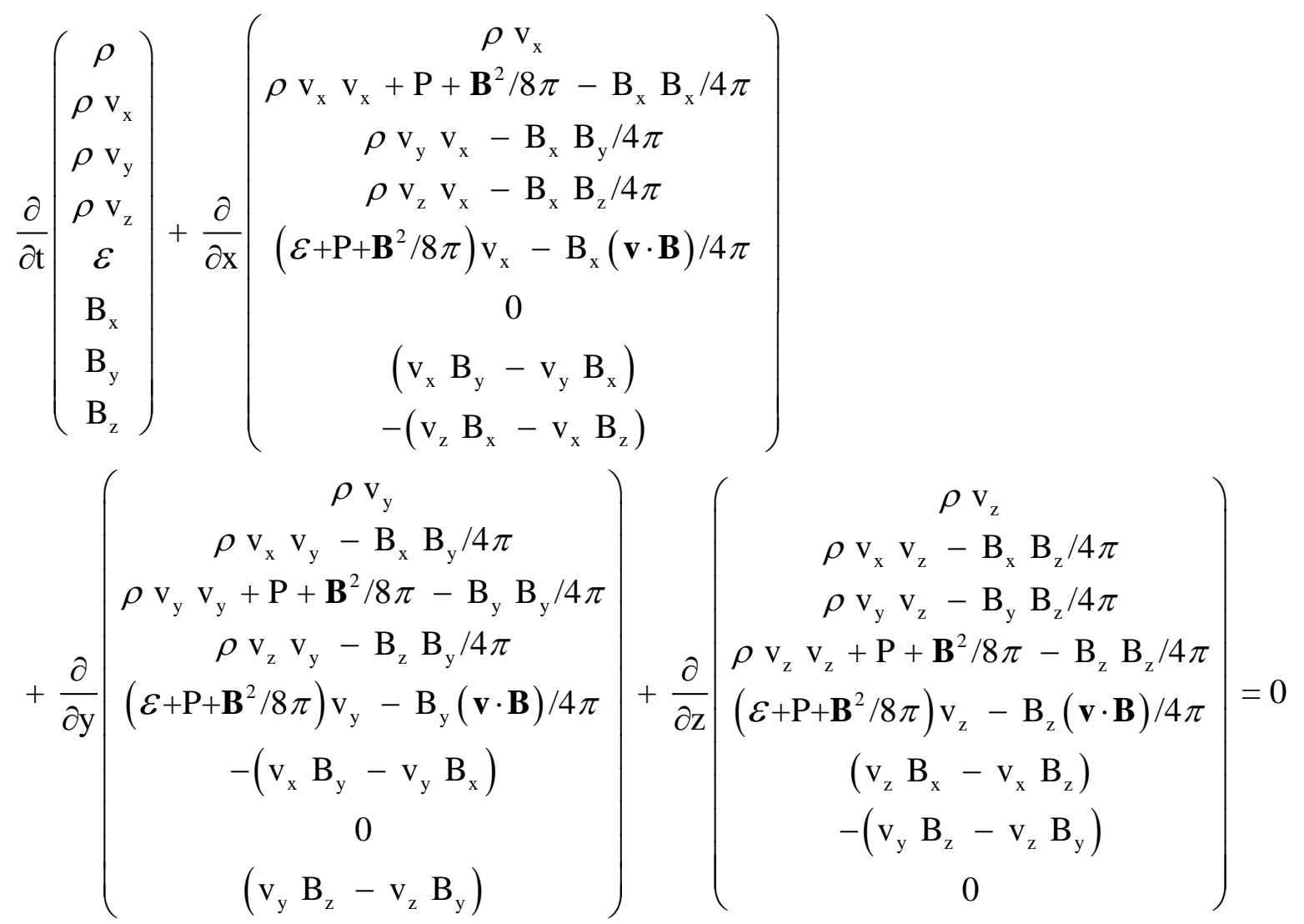

Here $\rho$ is the mass density, $\mathrm{P}$ is the pressure, $\varepsilon$ is the total energy density, $\mathrm{v}_{x}, \mathrm{v}_{y}, \mathrm{v}_{z}$ are the velocity components and $\mathrm{B}_{x}, \mathrm{~B}_{y}, \mathrm{~B}_{z}$ are the components of the magnetic field. The pressure is related to the energy density via a constitutive relation for an ideal gas, 
$P=(\gamma-1)\left(\varepsilon-\rho \mathbf{v}^{2} / 2-\mathbf{B}^{2} /(8 \pi)\right)$. The electric field components are given by $\mathrm{E}_{x}=-\left(\mathrm{v}_{y} \mathrm{~B}_{z}-\mathrm{v}_{z} \mathrm{~B}_{y}\right), \mathrm{E}_{y}=-\left(\mathrm{v}_{z} \mathrm{~B}_{x}-\mathrm{v}_{x} \mathrm{~B}_{z}\right)$ and $\mathrm{E}_{z}=-\left(\mathrm{v}_{x} \mathrm{~B}_{y}-\mathrm{v}_{y} \mathrm{~B}_{x}\right)$. The $\mathrm{x}-$ flux in eqn. (1.2) governs the propagation of waves in the $\mathrm{x}$-direction while the $\mathrm{y}$-flux governs the propagation of waves in the y-direction. From these equations we see that it is appropriate to obtain the $\mathrm{z}$ component of the electric field from the seventh component of the $\mathrm{x}$-flux when the waves (or shocks) are moving predominantly in the x-direction. By the same token, it is also appropriate to obtain the z-component of the electric field from the sixth component of the y-flux when the waves (or shocks) are moving predominantly in the y-direction. But for wave motions that are moving in an arbitrary direction, the one-dimensional Riemann solvers do not give proper guidance on which upwinded flux one has to choose in order to obtain the z-component of the electric field. Similar considerations apply to other components of the electric field.

Early thinking on this topic led to the suggestion that the dissipation from each direction should be doubled when evaluating the electric fields at the edges of the mesh (Gardiner \& Stone [43], [44]). A gedankenexperiment-type question that is worth asking is: Why should the dissipation always be doubled? Realize that there are indeed one-dimensional Riemann problems in each of the faces that come together at an edge. However, at each edge of the mesh the one-dimensional Riemann problems interact strongly with one another. This results in a strongly-interacting state which in most cases overlies the edge. The desired state and corresponding numerical fluxes that prevail at an edge are the ones associated with this stronglyinteracting state. Simple numerical experiments for the Euler equations of compressible gasdynamics by Schulz-Rinne, Collins \& Glaz [64] used one-dimensional Riemann solver technology to confirm that such a state indeed exists and evolves self-similarly as a function of time. In Balsara [15], [16], [19] we showed that the structure of the strongly-interacting state can be obtained by applying principles of self-similarity, entropy-enforcement and consistency with the multidimensional conservation law. These results were extended to unstructured meshes in Balsara, Dumbser \& Abgrall [17] and Balsara \& Dumbser [19]. It was also shown that the strongly-interacting state could be endowed with sub-structure, resulting in reduced dissipation (Balsara [16], [19], Balsara, Dumbser \& Abgrall [17], Balsara \& Dumbser [19]). The multidimensional Riemann solver designed in these works was given the name of MuSIC Riemann solver in Balsara [19]. The acronym stands for Multidimensional, Self-similar, strongly-Interacting state that is Consistent with the hyperbolic system. Further explanation for this acronym follows in the next few sentences. The multidimensionality of the MuSIC Riemann solver is self-evident, especially because it focuses on the strongly-interacting state that only occurs in multiple dimensions. The self-similarity pertains to the fact that the wave model used in such multidimensional Riemann solvers evolves self-similarly in space and time. That selfsimilarity has also been used to cast the conservation law in similarity variables which leads one to an evaluation of fluxes that is truly consistent with the hyperbolic system. (For a video introduction to multidimensional Riemann solvers, please see http://www.nd.edu/ dbalsara/Numerical-PDE-Course.) Application of the MuSIC Riemann 
solvers to divergence-free structured mesh MHD problems has shown that they work very well (Balsara [16], [18]). If one uses the family of MuSIC Riemann solvers, the doubling of dissipation from Gardiner \& Stone [43], [44] was not necessary any more. In addition, it was found that even for other systems, like the Euler equations, the MuSIC Riemann solvers provide greater isotropy in shock propagation and better preservation of contact discontinuities. They also permit larger CFL numbers to be used. The first goal of this work is to apply the MuSIC Riemann solvers to divergence-free MHD on unstructured meshes in two and three space dimensions. We wish to show that the advantages which were found for structured meshes carry over to unstructured meshes. The advantages include larger timesteps, more isotropic propagation of flow features and obviating the need to double the dissipation in the electric field. While the algorithms in this paper are explained for triangular elements in two space dimensions and tetrahedral elements in three space dimensions, the work is general and in principle extends also to other types of unstructured meshes.

Because MuSIC Riemann solvers are rather new, it helps to give further background information. Such multidimensional Riemann solvers act at the vertices of the mesh, where the multidimensional flow structure becomes visible to the Riemann solver. Instead of two input states, the input states consist of states from all the zones that meet at that vertex. At any zone interface that separates two states, a one dimensional Riemann problem emanates, as always. However, at any vertex, all the adjacent one-dimensional Riemann problems interact to form a strongly interacting state. The strongly interacting state evolves self-similarly in spacetime. By evolving the structure of the strongly interacting state in a set of self-similar variables we show that the structure of the strongly interacting state can be elucidated.

Implementation-related details for multidimensional HLLC-type Riemann solvers are given in Section IV of Balsara [16] or Section IV of Balsara, Dumbser \& Abgrall [17]. Implementation-related details for multidimensional Riemann solvers in self-similarity variables are given in Section VI of Balsara [18] or Section V of Balsara \& Dumbser [19]. Section III of Balsara [16] gives details that are specific to MHD. In particular, eqns. (34) and (35) from Section III of Balsara [16] explain how to obtain edge-centered electric fields from the multidimensional Riemann solver. Consequently, if a two-dimensional Riemann solver is invoked at the edges of the mesh, we will naturally retrieve the electric field component along that edge. The facially-averaged fluxes for conserved variables are obtained by a higher order quadrature formula that uses contributions from the one-dimensional and multidimensional Riemann solvers. Please see eqns. (24) and (25) from Balsara [15] for further details regarding facially-averaged fluxes.

Second order Godunov schemes for MHD that explicitly incorporate the divergence-free reconstruction of the magnetic field have been presented in Balsara [7], [8]. At second order, and on structured meshes without adaptive mesh refinement (AMR), the need for designing an appropriate divergence-free reconstruction for the magnetic field is not very compelling. At higher orders, one has to pay careful attention to the divergence-free aspect of the magnetic field 
(Balsara [9], Balsara et al. [12], [13], [14], Cockburn et al. [28], Li \& Shu [52], Li et al. [51], Christlieb et al. [27]). All of the above-mentioned advances have been made for structured meshes. A recent preprint ( $\mathrm{Xu}$ et al. [73]) has extended these advances to second and third orders on two-dimensional unstructured meshes. However, the formulation presented in the previous reference is quite cumbersome and it would be challenging to extend it to three-dimensions or very high order. In this paper we present a vastly simpler formulation that extends naturally to all orders on unstructured meshes in two- and three-dimensions. While various known schemes offer pathways to higher order accuracy, we prefer Weighted Essentially Non-Oscillatory (WENO) schemes because the method has an extensive and well-established literature (Shu \& Osher [66], [67], Abgrall [1], Jiang \& Shu [48], Balsara \& Shu [11], Balsara et al. [13], Dumbser \& Käser [39], Jiang \& Wu [49], Hu \& Shu [46]). The second goal of this work is to show that two- and three-dimensional globally divergence-free WENO schemes can be constructed for the magnetic field on unstructured meshes. Our formulation has been greatly influenced by the simplicity of the WENO algorithm of Dumbser \& Käser [39] and that algorithm will play a prominent role in the design of the divergence-free scheme that is presented here. When invoking this algorithm in the rest of this paper, we will refer to it as the "classical WENO" algorithm because it is a rather simple zone-based unstructured WENO algorithm that can be easily implemented in an unstructured mesh fluid flow code with higher order reconstruction.

As the spatial order of accuracy is increased, one should also increase the temporal order of accuracy. Runge-Kutta methods (Shu \& Osher [66], [67]) provide one strategy for increasing the temporal accuracy of any numerical method that has high spatial accuracy. However, the multi-stage nature of Runge-Kutta schemes makes them inconvenient for use in adaptive mesh refinement applications (Balsara [7], Dumbser et al. [38]). Consequently, one-step time-updates are favored (Dumbser et al. [36]). The so called Arbitrary DERivatives in space and time (ADER) methods provide such a time-stepping advantage (Titarev \& Toro [70], [71], Toro \& Titarev [72], Dumbser et al. [36], [37], Balsara et al. [13]). ADER schemes for MHD were first presented by Taube et al. [68]. The computational complexity of Runge-Kutta and ADER schemes have been cross-compared (Balsara et al. [14]) and the latter have been found to be superior. It is not the goal of this paper to design a new ADER scheme. However, a smaller third goal of this paper is to demonstrate that ADER-WENO schemes working with MuSIC Riemann solvers give us an effective strategy for high order divergence-free finite volume schemes for MHD on two- and three-dimensional unstructured meshes.

Please note that the formulation in this paper is a fundamentally novel departure from the way in which divergence-free ADER-WENO schemes for MHD have been designed and implemented in the past (Balsara et al. [13],[14]). In order to appreciate the differences, we provide Fig 1a which provides a schematic diagram of how a conventional divergence-free ADER-WENO schemes for MHD is implemented. Fig. 1b provides a schematic diagram of how the new divergence-free ADER-WENO scheme for MHD is implemented. First let us address Fig. 1a in this paragraph. In the next paragraph we will compare and contrast it with Fig. 1b. 
Please focus on Fig. 1a. It is broken up into four steps - reconstruction; predictor step; corrector step and update. In Fig. 1a we only evolve the zone-centered fluid variables (mass, momentum and energy densities) and face-centered components of the magnetic field. (Please note that "face-centered components of the magnetic field" refers to the normal components of the magnetic field vector in each face. In this paper we distinguish between a vector, which always has three components, and a vector component, which only refers to a single number.) Imagine, therefore, that a previous update step has used facially-averaged fluxes of fluid variables to update the zone-centered fluid variables. Likewise, the same update step has used edge-averaged electric fields to update the face-centered magnetic field components. The reconstruction step now produces the traditional WENO reconstruction of the fluid variables. It also produces the higher moments of the magnetic field components within each face. To carry out a predictor step within a zone, we need all of the spatial variation of all of the fluid plus magnetic field variables. (Variation just refers to the higher order modes that are needed for higher order accuracy. For zone-centered fluid variables, these are just the modes provided by classical, zone-centered WENO reconstruction.) We have the spatial variation of all of the fluid variables within the zone, but we have to build the spatial variation of the magnetic field variables within the entire zone in order to design an MHD predictor step. In Balsara [9] we present a strategy for using all the face-centered magnetic field information (face-averages and higher order moments) to obtain a divergence-free spatial representation of the magnetic field within a zone to sufficiently high order. The ADER predictor step then takes over. It takes the spatial information within a zone and, with the help of the governing PDE, produces a space and time evolution of the PDE within each zone. The corrector step then invokes face-centered one-dimensional Riemann solvers as well as edge-centered two-dimensional Riemann solvers to obtain facially-averaged fluid fluxes and edge-averaged electric field components. We are now ready for another update step.

The algorithm described here is a little more complicated, but its eventual implementation on unstructured meshes makes it vastly simpler and more practical. Please focus on Fig. $1 \mathrm{~b}$ now and please keep comparing it with Fig. 1a. The fundamental difference is that we retain the zone-averaged magnetic field vector as an auxiliary or helping variable. We hasten to add that these zone-averaged magnetic fields will be reset in the course of a timestep with the help of the correct, divergence-free, face-centered magnetic field components. The update step is mostly unchanged. The only difference is that the facially-averaged fluxes that could update the auxiliary zone-averaged magnetic field variables are also retained. As a result, the auxiliary magnetic field variables are also updated in the update step. This gives us an accurate set of auxiliary zone-averaged magnetic field variables which can now be used for making a classical WENO reconstruction of the magnetic field within a zone. This reconstruction is in general not divergence-free. But it is still sufficiently high order accurate. From this, the higher order moments of the magnetic field components can be evaluated within each face. Subsequently, using a novel constrained L2 projection technique, we utilize all the magnetic field information in the faces (face-averages and higher order moments) to modify the classical WENO reconstruction of the magnetic field so that it becomes locally and globally divergence-free and 
consistent with the facial information. This process also resets the zone-averaged magnetic field vector, making it consistent with the facial magnetic field information at each timestep. Because of this invention, we are able to rely on the well-known classical zone-based WENO reconstruction, which is subsequently post-processed by the new constrained L2 projection. This results in a considerable simplification of the algorithm, since it completely avoids the cumbersome face-based WENO reconstruction that was necessary in [73]. The zone-centered fluid variables are still reconstructed with classical WENO reconstruction. The ADER predictor step is unchanged. The corrector step, which involves one- and two-dimensional Riemann solvers is mostly unchanged. The only difference is that we store the facially-averaged magnetic fluxes along with the facially-averaged fluid fluxes. This enables us to update the auxiliary zoneaveraged magnetic variables in the update step. In the rest of this paper we will amplify on the algorithm that we have sketched our here. The reader is welcomed to keep revisiting Fig. 1b while reading the paper.

Please realize that the predictor, corrector and update steps are mostly unchanged in our new algorithm for divergence-free MHD. Thus the real focus of this paper is on a careful reworking of the classical, finite volume WENO algorithm to adapt it so that it can work on Yeetype meshes. Taken in that sense, this paper not only extends WENO to divergence-free MHD on unstructured meshes, but it also goes much further. The higher order WENO reconstruction reported here can touch all of computational electromagnetics. The other major focus of this paper is to show that multidimensional Riemann solvers play a crucial role in all divergence-free strategies applied to Yee-type meshes. Thus innovations made in MuSIC Riemann solvers are broad enough that they should also touch all of computational electromagnetics. While the focus of this paper is on WENO schemes, it is also realized that all finite volume type methods, like Discontinuous Galerkin and PnPm methods, will also benefit from this work.

The plan of the paper is as follows. In Section II we describe how the problem is set up and how the MuSIC Riemann solver is to be used for the time-update of the facial magnetic field components. In Section III we describe the role of zone-averaged auxiliary magnetic fields within each zone and show how they help in keeping the WENO reconstruction simple. In Section IV we describe how the results from the previous section are to be used to produce a divergence-free reconstruction of the magnetic field. Section $\mathrm{V}$ shows how the process of obtaining a divergence-free magnetic field can be automated on a computer at all orders using a constrained $\mathrm{L}_{2}$ projection method. Section VI presents a step-by-step synopsis of the algorithm that is suitable for computer implementation. Section VII presents accuracy analysis in two- and three-dimensions, showing that the schemes meet their design accuracy. Section VIII presents several stringent test problems. Section IX presents conclusions. 


\section{II) Problem Setup and Use of MuSIC Riemann Solver for Updating Magnetic Fields}

We divide this section into two parts. In Sub-section II.a we describe the problem setup when triangular elements are used in two-dimensions. In Sub-section II.b we describe the problem setup when tetrahedral elements are used in three-dimensions.

\section{II.a) Problem Setup and Use of MuSIC Riemann Solver on Triangular Elements}

The mesh is initialized by endowing each face of each triangle with a unit normal vector. Please see $\Delta P_{1} P_{2} P_{3}$ in Fig. 2. The unit normal vector does not have to be outward-pointing relative to any triangular element, because we will presently describe a strategy for obtaining the outward pointing normal relative to any triangle. Fig. 2 also shows that the lengths of the faces are given by $L_{1}, L_{2}$ and $L_{3}$. If the coordinates of the vertices of the triangle in question are known, the lengths of the faces are easily found. The unit normal vectors can be written in component form. For example, for $\mathbf{n}_{1}=\left(n_{1 x}, n_{1 y}\right)$ we can write

$$
n_{1 x}=\frac{y_{2}-y_{1}}{L_{1}} ; n_{1 y}=-\frac{x_{2}-x_{1}}{L_{1}}
$$

This allows us to define a parametric coordinate, $l_{1}$, in the segment $P_{1} P_{2}$ such that $l_{1} \in\left[-L_{1} / 2, L_{1} / 2\right]$. The coordinates of the segment $P_{1} P_{2}$ can be parameterized as

$$
\begin{aligned}
& x\left(l_{1}\right)=x_{C 1}-n_{1 y} l_{1} \quad ; \quad y\left(l_{1}\right)=y_{C 1}+n_{1 x} l_{1} ; \\
& \text { where } \quad x_{C 1}=\left(x_{1}+x_{2}\right) / 2 \quad \text { and } \quad y_{C 1}=\left(y_{1}+y_{2}\right) / 2
\end{aligned}
$$

Here $\left(x_{C 1}, y_{C 1}\right)$ is the midpoint of the segment $P_{1} P_{2}$. The normal component of the magnetic field in face $P_{1} P_{2}$ at any time $t^{n}$ can then be defined by

$$
\bar{B}_{1}^{n} \equiv \frac{1}{L_{1}} \int_{-L_{1} / 2}^{L_{1} / 2} \mathbf{B}\left(x\left(l_{1}\right), y\left(l_{1}\right), t^{n}\right)\left[\mathbf{n}_{1} d l_{1}\right.
$$

Here $\mathbf{B}(x, y, t)$ is a divergence-free magnetic field vector at the space-time point $(x, y, t)$. Notice that $\bar{B}_{1}^{n}$ is just the line-averaged value of the magnetic field component integrated over the segment $P_{1} P_{2}$. In this fashion, the magnetic field components can be defined at all the faces of the triangular elements. Eqn. (2.3) serves to define the magnetic field component at any face of the triangle. These facially-averaged magnetic field variables are the evolutionary variables of our divergence-free MHD scheme. Please note that only the normal components of the magnetic field are defined at the zone-faces. I.e., we distinguish between the magnetic field component 
and the entire magnetic field vector. In the rest of this paper we will meticulously refer to the facially-averaged magnetic field components, which are given by specifying one numerical value in each of the faces of each element. We will also feel a need to refer to the magnetic field, which consists of a vector that is specified at each point in space.

When implementing this algorithm it would help if one can check that the magnetic field components that have been initialized at the zone faces are indeed divergence-free on an element-by-element basis. To do that, we need the concept of an outward-pointing unit normal relative to an element. In the following we denote the simplex control volumes ( triangles in twodimensions and tetrahedra in three-dimensions) by $T_{i}$ and the faces (line segments in twodimensions and triangles in three-dimensions) with $F_{j}$. The set of faces that defines the control volume $T_{i}$ is denoted by $\mathrm{F}_{i}$. Then the outward pointing unit normal vector of a face $F_{j} \in \mathrm{F}_{i}$ relative to element $T_{i}$ is denoted by $\mathbf{n}_{i j}=\sigma_{i j} \cdot \mathbf{n}_{j}$, where the sign function $\sigma_{i j}$ is given by $\sigma_{i j}=\operatorname{sign}\left(\left(\mathbf{x}_{j}-\mathbf{x}_{i}\right) \cdot \mathbf{n}_{j}\right)$. Here, $\mathbf{x}_{i}$ and $\mathbf{x}_{j}$ denote the barycenter of the control volume $T_{i}$ and of the face $F_{j}$, respectively. With that convention, at any time $t^{n}$ the discrete divergence-free condition for the edge-averaged normal components of the magnetic field for a general element $T_{i}$, like the one sketched in Fig. 2, can be written as

$$
\sum_{j \in \mathrm{F}_{i}} \sigma_{i j} \bar{B}_{j}^{n} L_{j}=0, \quad \forall T_{i}
$$

It might also be useful to check that the previous identity is satisfied up to machine accuracy at various times during the course of a simulation. We also make an implementation-related suggestion. Each control volume $T_{i}$ only needs to retain one bit of information for each of its faces ( 3 faces in two-dimensions and 4 faces in three-dimensions) that tell it whether the unit normal vector in each of its three faces is outward- or inward-pointing, in order to store the sign function $\sigma_{i j}$.With just this little extra information, one can evaluate eqn. (2.4). As we can see, the memory overhead and increased computational complexity for supporting a divergence-free formulation is minimal. The calculation just needs to be set up properly.

In Fig. 2, all the states that come together at a vertex can be provided to the MuSIC Riemann solver. The multidimensional MuSIC Riemann solver will, in turn, produce a $\mathrm{z}$ component of the electric field which can be used to update the three magnetic field components in the faces of the element. For two-dimensional calculations, it is best to retain the convention that the z-component of the magnetic field points out of the plane of the paper shown in Fig. 2. Let $\left\langle E_{z 1}^{n+1 / 2}\right\rangle,\left\langle E_{z 2}^{n+1 / 2}\right\rangle$ and $\left\langle E_{z 3}^{n+1 / 2}\right\rangle$ be the time-averaged electric field components at the vertices 
$P_{1}, P_{2}$ and $P_{3}$ of triangle $T_{i}$ depicted in Fig. 2. Then, the time-update from time $t^{n}$ to time $t^{n+1}=t^{n}+\Delta t$ for the magnetic field components can be written as:

$$
\begin{aligned}
& \bar{B}_{1}^{n+1}=\bar{B}_{1}^{n}-\Delta t \frac{\left\langle E_{z 2}^{n+1 / 2}\right\rangle-\left\langle E_{z 1}^{n+1 / 2}\right\rangle}{L_{1}} ; \\
& \bar{B}_{2}^{n+1}=\bar{B}_{2}^{n}-\Delta t \frac{\left\langle E_{z 3}^{n+1 / 2}\right\rangle-\left\langle E_{z 2}^{n+1 / 2}\right\rangle}{L_{2}} ; \\
& \bar{B}_{3}^{n+1}=\bar{B}_{3}^{n}-\Delta t \frac{\left\langle E_{z 1}^{n+1 / 2}\right\rangle-\left\langle E_{z 3}^{n+1 / 2}\right\rangle}{L_{3}}
\end{aligned}
$$

It is very easy to show that if the divergence-free condition in eqn. (2.4) is satisfied at time $t^{n}$ then it will also be satisfied at time $t^{n+1}$. The update in eqn. (2.5a) is easily implemented by visiting each face $F_{j}$ and using the z-components of the electric field computed by the MuSIC Riemann solver on the left and right node of the oriented face $F_{j}$ as follows:

$$
\bar{B}_{j}^{n+1}=\bar{B}_{j}^{n}-\Delta t \frac{\left\langle E_{z, r}^{n+1 / 2}\right\rangle-\left\langle E_{z, l}^{n+1 / 2}\right\rangle}{L_{j}},
$$

where $l=l(j)$ denotes the number of the left node $P_{l}$ and $r=r(j)$ the number of the right node $P_{r}$ of the oriented face $F_{j}$. Since $E_{z}$ can be either obtained from the seventh component of the Xflux of the MHD equations or from the sixth component of the y-flux, we simply take an arithmetic average of the two to define $E_{z}$ uniquely in eqn. (2.5b). This completes our description of the divergence-free time update of the face-averaged magnetic fields on twodimensional unstructured meshes.

In $\mathrm{Xu}$ et al. [73] a divergence-free algorithm was presented that only relied on the magnetic field components at zone faces. I.e., the zone-centered magnetic field plays no part in the timestep of that algorithm. Making that choice also meant that one has to use two different reconstruction steps: the first being defined on edge-based stencils, like the one used in [21] for the high order reconstruction of velocity fields from face-averaged normal velocity components on general unstructured meshes, and the second one using cell-based stencils, as the unstructured WENO finite volume reconstruction proposed in [39]. On an unstructured mesh, this can slow down the computation. The goal of this paper is to dramatically simplify the high order divergence-free reconstruction procedure for the magnetic field. For that, we retain the zoneaveraged magnetic field in each zone as an auxiliary (or helping) variable. However, an intricate rearrangement of the algorithm is used that enables us to overwrite the zone-averaged magnetic field at the start of each timestep by using the facially-averaged magnetic field components. The 
detailed strategy for doing this is described in Section III and mathematically prescribed in Section IV. In Section V we show how the same strategy can be automated for use in computer code at all orders of accuracy. We just state here that after the zone-centered WENO reconstruction step and before the start of each ADER predictor step we overwrite the zoneaveraged magnetic field at the center of the triangular zone shown in Fig. 2 given by $\mathbf{B}_{i}^{n}$. The motivation for arranging the algorithm this way is also presented in Section III.

It is also useful to introduce the reader to some of the issues involved in making a divergence-free reconstruction of the magnetic field. At a time $t^{n}$ we start with the faciallyaveraged magnetic field components, see eqn. (2.3). Say we want fourth order accurate reconstruction. By the time the facial reconstruction is completed, we want the magnetic field component in the segment $P_{1} P_{2}$ of Fig. 2 to have spatial variation given by

$$
B_{1}^{n}(\xi)=\bar{B}_{1}^{n}+\left(\partial_{\xi} B_{1}^{n}\right) \xi+\frac{1}{2}\left(\partial_{\xi}^{2} B_{1}^{n}\right)\left(\xi^{2}-\frac{L_{1}^{2}}{12}\right)+\frac{1}{6}\left(\partial_{\xi}^{3} B_{1}^{n}\right)\left(\xi^{3}-\frac{3 L_{1}^{2}}{20} \xi\right)
$$

In the above equation, $\xi$ is a local coordinate in the face being considered and has the range $\left[-L_{1} / 2, L_{1} / 2\right]$. Notice that the use of the basis polynomials above ensures that the mean value of the magnetic field in the face is preserved. In practice, other kinds of orthogonal modal basis polynomials can also be used, like the classical Legendre polynomials. The first step in the reconstruction process consists of obtaining the first, second and third moments of eqn. (2.6).(The number of moments that are retained. In eqn. (2.6) depends on the desired order.) In other words, we wish to obtain $\left(\partial_{\xi} B_{1}^{n}\right),\left(\partial_{\xi}^{2} B_{1}^{n}\right)$ and $\left(\partial_{\xi}^{3} B_{1}^{n}\right)$ respectively, as needed. Section III and Sub-section IV.a will describe how this is done. With the use of appropriate basis polynomials, eqn. (2.6) also illuminates the path to even higher orders. Equations that are analogous to eqn. (2.6) can be obtained for all the faces of the triangular element shown in Fig. 2.

Realize now that the first step from the previous paragraph only gives us the magnetic field component and its higher order moments in each of the three faces of a triangle. In order to provide input states to a Riemann solver, we want the magnetic field to be suitably reconstructed all over the zone. Furthermore, it helps if that reconstructed magnetic field is divergence-free everywhere within the triangular zone shown in Fig. 2. Such a divergence-free magnetic field will exactly match the magnetic field component and their variation within the faces of the triangles; thereby avoiding the formation of magnetic monopoles on the mesh. This is the second step in the reconstruction process and will be thoroughly described in Sub-section IV.a. Section $\mathrm{V}$ will subsequently show how this procedure can be automated on a computer at all orders.

\section{II.b) Problem Setup and Use of MuSIC Riemann Solver for Tetrahedral Elements}


The left panel from Fig. 3 shows an element from a tetrahedral mesh. Each triangular face has a unit normal vector. As before, the unit normal vector does not have to be outwardpointing, because we will soon describe a strategy for obtaining the unit outward-pointing normal for each tetrahedron. The unit normal vector can be written in component form. For example, for $\mathbf{n}_{1}=\left(n_{1 x}, n_{1 y}, n_{1 z}\right)$ we can write

$$
\mathbf{n}_{1}=\frac{\overrightarrow{P_{1} P_{2}} \times \overrightarrow{P_{1} P_{3}}}{\left|\overrightarrow{P_{1} P_{2}} \times \overrightarrow{P_{1} P_{3}}\right|}
$$

The right panel from Fig. 3 shows that within each face, we can also define two unit vectors that reside entirely within that face. Notice that the unit vectors $\xi_{1}, \boldsymbol{\eta}_{1}$ and $\mathbf{n}_{1}$ in $\Delta P_{1} P_{2} P_{3}$ form a right-handed coordinate system. In component form we can write $\xi_{1}=\left(\xi_{1 x}, \xi_{1 y}, \xi_{1 z}\right)$ and $\boldsymbol{\eta}_{1}=\left(\eta_{1 x}, \eta_{1 y}, \eta_{1 z}\right)$ and distances measured along those two vectors constitute a local coordinate system, $(\xi, \eta)$, within $\Delta P_{1} P_{2} P_{3}$. Let $C_{1} \equiv\left(x_{C 1}, y_{C 1}, z_{C 1}\right)$ denote the centroid of the $\Delta P_{1} P_{2} P_{3}$ and let the $(\xi, \eta)$ coordinate system within this triangle be centered at the centroid. The area of the $\Delta P_{1} P_{2} P_{3}$ is then parameterized by

$$
x(\xi, \eta)=x_{C 1}+\xi_{1 x} \xi+\eta_{1 x} \eta ; y(\xi, \eta)=y_{C 1}+\xi_{1 y} \xi+\eta_{1 y} \eta ; z(\xi, \eta)=z_{C 1}+\xi_{1 z} \xi+\eta_{1 z} \eta
$$

The facially-averaged normal component of the magnetic field in $\Delta P_{1} P_{2} P_{3}$ at time $t^{n}$ can then be defined by

$$
\left.\bar{B}_{1}^{n} \equiv \frac{1}{A_{1}} \int_{\Delta P_{1} P_{2} P_{3}} \mathbf{B}\left(x(\xi, \eta), y(\xi, \eta), z(\xi, \eta), t^{n}\right)\right] \mathbf{n}_{1} d \xi d \eta
$$

Here $A_{1}$ is the area of $\Delta P_{1} P_{2} P_{3}$ and $\mathbf{B}(x, y, z, t)$ is a divergence-free magnetic field vector at the space-time point $(x, y, z, t)$. Notice that $\bar{B}_{1}^{n}$ is the area-averaged magnetic field component integrated over $\Delta P_{1} P_{2} P_{3}$. In this fashion, the magnetic field components can be defined at all the faces of the tetrahedral elements. Eqn. (2.9) serves to define the magnetic field component at any face of the tetrahedron. These facially-averaged magnetic field variables are the evolutionary variables of our divergence-free MHD scheme. In practice, a magnetic vector potential is used to initialize a divergence-free magnetic field at the faces of the computational mesh, see Balsara [8] for more details.

When implementing this algorithm it would help if one can check that the magnetic field components that have been initialized at the zone faces are indeed divergence-free also at the discrete level. To do that we use the same sign function $\sigma_{i j}$ as defined before for triangular 
elements in two space dimensions. With that convention, the discrete divergence-free condition at any time $t^{n}$ for the face-averaged normal components of the magnetic field on the tetrahedron shown in Fig. 3 can be written as

$$
\sigma_{i 1} \bar{B}_{1}^{n} A_{1}+\sigma_{i 2} \bar{B}_{2}^{n} A_{2}+\sigma_{i 3} \bar{B}_{3}^{n} A_{3}+\sigma_{i 4} \bar{B}_{4}^{n} A_{4}=0
$$

The multidimensional MuSIC Riemann solver is designed to work in two dimensions. It returns two fluxes in the two-dimensional plane within which it is invoked. Those two fluxes can also be used to yield the electric field component in a direction that is orthogonal to the plane in which the multidimensional Riemann solver is invoked. For that reason, in Fig. 3, we specify a unit vector, generically called $\mathbf{e}_{k}$ within each edge $E_{k}$ of the tetrahedron. The update of the magnetic field component within each face takes place in the following three steps:

1) Within each edge, visit all the zones around that edge and collect all the corresponding states for input to the MuSIC Riemann solver. Since the MuSIC Riemann solver accepts all input states with a counterclockwise orientation (Balsara\&Dumbser [19]) it helps that each edge in the left panel of Fig. 3 has a pre-specified orientation. From the output of the MuSIC Riemann solver, we can obtain all the fluxes and electric fields at that edge. The fluxes can then be distributed to all the participating faces of the mesh. The electric field component is stored at the edge. This operation loops over all the edges of the mesh.

2) For each oriented face $F_{j}$ we first pick the corresponding unit normal $\mathbf{n}_{j}$. For each edge $E_{k}$ spanned by the unit vector unit vector $\mathbf{e}_{k}$ we define a sign function $\sigma_{j k}^{\prime}$ that has the value +1 if the edge is oriented counterclockwise relative to the outward pointing unit normal, otherwise it has the value -1 . The right panel in Fig. 3 shows such an example where the unit vectors in all the edges have a counter-clockwise orientation relative to the unit outward-pointing normal $\mathbf{n}_{1}$.

3) Let $\left\langle E_{k}^{n+1 / 2}\right\rangle$ be the electric field component that has been averaged over one space and one time direction along the unit vector $\mathbf{e}_{k}$, see the right panel of Fig. 3. The spatial averaging of those electric field components are done along the edges that they belong to. The time-averaging is done from time $t^{n}$ to time $t^{n+1}=t^{n}+\Delta t$. At higher orders, this space-time averaging turns into a suitable quadrature in space and time. If we denote the set of edges that constitutes the face $F_{j}$ by $\mathrm{E}_{j}$, then the temporal update of the magnetic field component within a face $F_{j}$ reads

$\bar{B}_{j}^{n+1}=\bar{B}_{j}^{n}-\frac{\Delta t}{A_{j}} \sum_{k \in \mathrm{E}_{j}} \sigma_{j k}^{\prime}\left\langle E_{k}^{n+1 / 2}\right\rangle L_{k}$

As a particular example, for the situation shown in the right panel of Fig. 3 we simply have 
$\bar{B}_{1}^{n+1}=\bar{B}_{1}^{n}-\frac{\Delta t}{A_{1}}\left(\left\langle E_{1}^{n+1 / 2}\right\rangle L_{1}+\left\langle E_{2}^{n+1 / 2}\right\rangle L_{2}+\left\langle E_{3}^{n+1 / 2}\right\rangle L_{3}\right)$

Proper application of this algorithm will yield a divergence-free update of the magnetic field on all the tetrahedral elements.

To implement the algorithm described in this paper, we need to obtain an adequately high order representation of the zone-averaged magnetic field within the tetrahedral zone shown in Fig. 3. This can be done by asserting that the zone-averaged magnetic field at the center of the triangle is given by $\mathbf{B}_{i}^{n}$ and is treated as an auxiliary variable. As with the two-dimensional case, these zone-averaged magnetic fields will be overwritten by using the facial magnetic field components after the WENO reconstruction but before the ADER predictor step. The detailed strategy for doing this is described in Section III and mathematically prescribed in Section IV. In Section V we show how the same strategy can be automated for use in computer code at all orders of accuracy.

We now wish to build the analogue of eqn. (2.6) in two-dimensions. In this context, realize that on a triangular face one should best use the orthogonal Dubiner basis polynomials given in [35],[65],[60], which is also what we have implemented in our computer code, but to give a quick and more concise overview of the basic ideas, we show simply Taylor monomials in the following. Before we do that, we need a quick strategy for evaluating the integral of $\xi^{p} \eta^{q}$ over a triangular face like the one shown in the right panel of Fig. 3. Here " $p$ " and " $q$ " are usually small non-negative integers. Such a strategy is presented in Section IV.a of Balsara \& Dumbser [19]. We denote the moments over $\Delta P_{1} P_{2} P_{3}$ with the following compact notation

$$
\begin{aligned}
& I_{\triangle P_{1} P_{2} P_{3}}^{0} \equiv \iint_{\triangle P_{1} P_{2} P_{3}} d \xi d \eta ; I_{\Delta P_{1} P_{2} P_{3}}^{\xi} \equiv \iint_{\triangle P_{1} P_{2} P_{3}} \xi d \eta d \eta ; \quad I_{\Delta P_{1} P_{2} P_{3}}^{\eta} \equiv \iint_{\triangle P_{1} P_{2} P_{3}} \eta d \xi d \eta ; \\
& I_{\Delta P_{1} P_{2} P_{3}}^{\xi \xi} \equiv \iint_{\Delta P_{1} P_{2} P_{3}} \xi \xi d \xi d \eta ; I_{\Delta P_{1} P_{2} P_{3}}^{\eta \eta_{3}} \equiv \iint_{\Delta P_{1} P_{2} P_{3}} \eta \eta d \xi d \eta ; I_{\Delta P_{1} P_{2} P_{3}}^{\xi \eta} \equiv \iint_{\Delta P_{1} P_{2} P_{3}} \xi \eta d \xi d \eta \text {; etc. }
\end{aligned}
$$

The analogue of eqn. (2.6) in two-dimensions is now given up to fourth order by

$$
\begin{aligned}
B_{1}^{n}(\xi, \eta)= & \bar{B}_{1}^{n}+\left(\partial_{\xi} B_{1}^{n}\right) \xi+\left(\partial_{\eta} B_{1}^{n}\right) \eta \\
& +\frac{1}{2}\left(\partial_{\xi \xi}^{2} B_{1}^{n}\right)\left(\xi^{2}-\frac{I_{\Delta P_{2} P_{2} P_{3}}^{\xi \xi}}{I_{\Delta P_{1} P_{2} P_{3}}^{0}}\right)+\left(\partial_{\xi \eta}^{2} B_{1}^{n}\right)\left(\xi \eta-\frac{I_{\Delta P_{1} P_{2} P_{3}}^{\xi \eta_{2}}}{I_{\Delta P_{1} P_{2} P_{3}}^{0}}\right)+\frac{1}{2}\left(\partial_{\eta \eta}^{2} B_{1}^{n}\right)\left(\eta^{2}-\frac{I_{\Delta P_{2} P_{2} P_{3}}^{\eta}}{I_{\Delta P_{1} P_{2} P_{3}}^{0}}\right) \\
& +\frac{1}{6}\left(\partial_{\xi \xi \xi}^{2} B_{1}^{n}\right)\left(\xi^{3}-\frac{I_{\Delta P_{1} P_{2} P_{3}}}{I_{\Delta P_{1} P_{2} P_{3}}^{0}}\right)+\frac{1}{2}\left(\partial_{\xi \xi \eta}^{2} B_{1}^{n}\right)\left(\xi \xi \eta-\frac{I_{\Delta P_{1} P_{2} P_{3}}}{I_{\Delta P_{1} P_{2} P_{3}}}\right)+\frac{1}{2}\left(\partial_{\xi \eta \eta}^{2} B_{1}^{n}\right)\left(\xi \eta \eta-\frac{I_{\Delta P_{1} P_{2} P_{3}}^{\xi \eta \eta}}{I_{\Delta P_{1} P_{2} P_{3}}^{0}}\right) \\
& +\frac{1}{6}\left(\partial_{\eta \eta \eta}^{2} B_{1}^{n}\right)\left(\eta^{3}-\frac{I_{\Delta P_{1} P_{2} P_{3}}^{\eta \eta n}}{I_{\Delta P_{1} P_{2} P_{3}}^{0}}\right)
\end{aligned}
$$


Please note that the coordinates $(\xi, \eta)$ are defined relative to the facial centroid $C_{1}$ in Fig. 3. The first line of eqn. (2.13) yields second order accuracy, consequently, specifying the coefficients $\left(\partial_{\xi} B_{1}^{n}\right)$ and $\left(\partial_{\eta} B_{1}^{n}\right)$ gives us second order accuracy. The second line of eqn. (2.13) gives third order of accuracy, consequently, third order accuracy requires us to specify the coefficients $\left(\partial_{\xi \xi}^{2} B_{1}^{n}\right),\left(\partial_{\xi \eta}^{2} B_{1}^{n}\right)$ and $\left(\partial_{\eta \eta}^{2} B_{1}^{n}\right)$. Analogously, the third and fourth lines of eqn. (2.13) give fourth order of accuracy. Section III and Sub-section IV.b will describe how these coefficients are specified. Note that the expansion basis functions shown in eqn. (2.13) are not orthogonal, but they do have the essential property that all the higher order basis functions have a zero average over the triangle. In our practical implementation, however, we have used the orthogonal Dubiner basis detailed in [35],[65],[60].

While specification of the coefficients in eqn. (2.13) is the first step in the divergencefree reconstruction, there is a further second step. Section IV.b will show us how the specification of the magnetic field components and their variation within each boundary will, in turn, yield a divergence-free reconstruction of the magnetic field vector within the entire interior volume of the tetrahedron in Fig. 3.

\section{III) A preview of the new method}

\section{III.a) Definition of divergence-free magnetic fields at the discrete level}

Before we start with the preview of our new numerical method and before motivating the introduction of a new auxiliary zone-averaged magnetic field, let us define what we exactly intend with divergence-free magnetic fields at the discrete level:

Definition 1: The face-averaged normal magnetic field components $\bar{B}_{j}^{n}$ are said to be exactly divergence free at the discrete level, if the integral relations (2.4) and (2.10) hold up to machine precision for all elements $T_{i}$ of the triangulation/tetrahedrization of the computational domain $\Omega$. This means that we require the integral identity

$$
\int_{\partial T_{i}} \mathbf{B}\left(\mathbf{x}, t^{n}\right) \cdot \mathbf{n} d S=0, \quad \forall T_{i} \in \Omega
$$

to be valid at the discrete level for all elements of the computatioanl domain $\Omega$.

Definition 2: A high order zone-centered piecewise polynomial reconstruction $\mathbf{B}_{h}^{n}\left(\mathbf{x}, t^{n}\right)$ of the magnetic field vector is said to be locally and globally divergence free at the discrete level, if the following two conditions hold: 
- The field is locally divergence free everywhere in the entire computational domain $\Omega$, i.e. the following relation must hold pointwise everywhere inside the computational domain up to machine precision:

$$
\nabla \cdot \mathbf{B}_{h}^{n}\left(\mathbf{x}, t^{n}\right)=0, \quad \forall \mathbf{x} \in \Omega
$$

- The normal component of the magnetic field is continuous across each face $F_{j}$, i.e.

$$
\mathbf{B}_{h}^{n}\left(\mathbf{x}^{-}, t^{n}\right) \cdot \mathbf{n}_{j}=\mathbf{B}_{h}^{n}\left(\mathbf{x}^{+}, t^{n}\right) \cdot \mathbf{n}_{j}, \quad \forall \mathbf{x} \in F_{j}
$$

where $\mathrm{x}^{-}$and $\mathrm{x}^{+}$denote the evaluation of the magnetic field from within the left and the right element attached to a face $\mathrm{F}_{j}$, respectively. It is condition (3.2) that ensures, together with (3.1), that a high order zone-centered polynomial reconstruction of the magnetic field can also be called globally divergence-free at the discrete level.

\section{III.b) The Role of the Auxiliary Zone-Averaged Magnetic Field}

Let us consider that the MHD computation has been evolved to a time $t^{n}$. In Section II, we mentioned that the simplicity of our method derives from the fact that we use zone-centered magnetic fields as helping/auxiliary variables in the reconstruction. Thus the zone-averaged magnetic field $\mathbf{B}_{i}^{n}$ is presumed to be available within each two-dimensional triangle or threedimensional tetrahedron at the start of a timestep. These are the variables that have been computed using a preliminary zone-centered ADER-WENO finite volume update for the cell average of the magnetic field vector. Do please note though, that they are never the primary variables for the magnetic field and, furthermore, that these preliminary cell averages are overwritten after the divergence-free reconstruction step with the help of the facial components of the magnetic fields. This is done via the following three-step strategy:

1) In the first step we apply the classical WENO reconstruction to all the zone-centered variables, including the zone-averaged, auxiliary magnetic fields. This gives us zone-centered reconstructing polynomials for the components of the zone-averaged magnetic field. These reconstructing polynomials for the magnetic field are in general not yet divergence free in the sense of conditions (3.1) and (3.2). They are, nevertheless, non-linearly hybridized by the classical WENO limiting.

2) The above-mentioned zone-centered reconstructing polynomials for the components of the zone-averaged magnetic field are then used to provide the higher order moments of the magnetic field components in the faces of each zone. I.e. see eqns. (2.6) and (2.12) in two- and threedimensions. The mean magnetic field component in each face is left untouched. Using a procedure that is described in detail in Sections IV and V we now build a divergence-free higher order reconstruction of the magnetic field within each zone. 
3) The divergence-free reconstructed polynomials for the magnetic field within each zone are now used to obtain a new zone-averaged magnetic field which overwrites the auxiliary zoneaveraged magnetic field.

Notice that this entire three-step strategy takes place at time $t^{n}$ before any ADER predictor step is applied. We explain why this strategy is very useful in the next few paragraphs.

Each timestep starts with the zone-centered fluid variables, the face-averaged magnetic field components as well as the auxiliary zone-averaged magnetic fields. Using the zonecentered fluid variables and the zone-averaged magnetic fields, we carry out the classical zonebased WENO reconstruction on unstructured meshes using the algorithm of Dumbser \& Käser [39]. Amongst other things, this gives us the variation of the magnetic field on either side of a face. Consequently, each face can provide higher order moments to its magnetic field component by using the zone-centered reconstruction that has been carried out in the two zones on either side of it. Typically, we take a minmod of the two higher order moments coming from either side.

Let us illustrate this in two-dimensions by focusing on eqn. (2.6) and Fig. 2. There will always be two triangles on either side of any interior zone boundary. The zone-centered classical WENO reconstruction, therefore, gives us two alternative ways of specifying the higher order moments in eqn. (2.6). We follow Xu et al. [73] and take the minmod function of the two choices. This fully specifies all the moments in eqn. (2.6). An entirely analogous procedure in three-dimensions will give us the higher order moments in eqn. (2.13). Note that in either eqn. (2.6) or eqn. (2.13) we leave the face-averaged magnetic field component, $\bar{B}_{1}^{n}$, untouched.

Once the moments of the facial variables are available, we make a higher order divergence-free reconstruction of the entire magnetic field within each zone using methods that are described in Section IV and automated for use on a computer in Section V. This divergencefree reconstruction is averaged over each zone to yield a new zone-averaged magnetic field. This zone-averaged magnetic field is high-order accurate, i.e. of an accuracy that is sufficient to match the accuracy of the rest of the scheme. It is also a natural byproduct of making a sufficiently accurate divergence-free reconstruction within a zone. We use that magnetic field to overwrite the auxiliary, zone-averaged magnetic field $\mathbf{B}_{i}^{n}$ in two- and three-dimensions. Section $\mathrm{V}$ shows exactly how this is done at all orders. This completes the higher order reconstruction step. Please notice that by overwriting the zone-averaged field, we ensure that the zone-averaged magnetic field variables are always well-coupled to the facial magnetic field components. It is also apparent now that the facial magnetic field components are the primary variables for the magnetic field.

We are now ready for the ADER predictor-corrector sequence. The spatial variation of the flow variables and the magnetic field are now in hand. The ADER predictor step from Dumbser et al. [36] yields its temporal extension. The ADER predictor step is always done in the 
zone because that is where the full spatial variation within a zone can be exploited, along with the structure of the PDE, to obtain the space-time extension of the flow variables and the magnetic field. The corrector step, which is based on Riemann solvers, can now be applied. In the corrector step we update the five flow variables in eqn. (1.2) as well as the auxiliary zonecentered magnetic field components. This is done using the traditional flux formulation from eqn. (1.2). We also use eqns. (2.5) or (2.11), as appropriate, to obtain the update of the faceaveraged magnetic field components. Please observe that by this point in the narrative the zone-

centered flow variables and facial magnetic field variables have reached a time $t^{n+1}$. However, since we updated all eight variables in eqn. (1.2), we also have the auxiliary zone averages of the magnetic field $\mathbf{B}_{i}^{n+1}$ in two- and three-dimensions. Furthermore, these are perfectly good higher order updates which can now be used to initiate the classical WENO reconstruction at time $t^{n+1}$. Thus the order property is preserved in our update.

The reason for overwriting the zone-averaged magnetic field in every timestep is as follows. In very long-running calculations, the zone-averaged magnetic field can slowly become disconnected from the facial magnetic field components if nothing is done. Since the facial magnetic field variables are the primary variables in our scheme, they should provide correction to the zone-centered magnetic field at each timestep. Our construction is such that the correction with sufficient order of accuracy is always provided. In that sense, the zone-averaged magnetic field is only an auxiliary variable, not a primary variable. It is only evolved for one timestep and then it is overwritten. Also notice that our primary magnetic field variables (the face-averaged magnetic field components) are divergence-free at a discrete level in the sense of equations (2.4) and (2.10), and they remain so throughout the entire simulation. As a result, any slight build-up of divergence in the zone-averaged magnetic field is negligible in the course of one timestep. After the zone-averaged magnetic field has helped us in making the classical WENO reconstruction within a zone, it will indeed be overwritten.

\section{III.c) Comparison with the algorithm proposed by $\mathrm{Xu}$ et al. in [73]}

As already mentioned before, the new method introduced in this paper has the aim to avoid the cumbersome face-based WENO reconstruction that is necessary to produce a high order polynomial reconstruction of the magnetic field from the known face-averaged magnetic field components. More precisely, the algorithm presented in [73] requires two completely different WENO reconstruction steps. The first one is a conventional zone-based WENO reconstruction that converts the zone-averaged fluid variables into high order zone-centered piecewise polynomial reconstructions. The second one is a face-based WENO reconstruction, which converts the face-averaged normal magnetic field components into a high order reconstruction polynomial.

By introducing the auxiliary zone-averaged magnetic field, we can completely avoid the second face-based WENO reconstruction step that was necessary in [73]. Instead, we can just perform 
one simple classical zone-based WENO reconstruction for all quantities, including fluid variables and magnetic field. The resulting high order piecewise polynomial reconstruction of the magnetic field is then made locally and globally divergence-free in the sense of conditions (3.1) and (3.2). This is achieved by using the information from the known face-averaged normal magnetic field components and the new constrained L2 projection technique detailed in Section V.

\section{IV) Divergence-free Reconstruction of the Magnetic Field}

The one-dimensional Riemann problem for MHD carries with it one essential subtlety (Jefferey\&Taniuti [47]): The normal component of the magnetic field has to be continuous across the Riemann problem in order for the Riemann problem to be well-defined. This is the very reason for requiring condition (3.2) to be satisfied for a high order polynomial reconstruction of the magnetic field. As a result, in two- and three-dimensions we want the coefficients of the magnetic field components in eqns. (2.6) and (2.13) to be uniquely specified at a zone-boundary.

Following Balsara [7], [8] and [9] it is possible to demand that the magnetic field should be divergence-free everywhere within a zone. Such a divergence-free magnetic field should coincide with the normal component of the magnetic field and its facial variation within the faces of each element. There is much merit in such an enterprise. Most importantly, it ensures that the one-dimensional Riemann problem for MHD remains well-defined. Additionally, it can open the door to divergence-free adaptive mesh refinement for MHD on unstructured meshes. (Please see Balsara [7] for an example of divergence-free adaptive mesh refinement for MHD on structured meshes.) For that reason, the next two sub-sections provide details about the divergence-free reconstruction of the magnetic field on unstructured meshes.

\section{IV.a) Divergence-free Reconstruction of the Magnetic Field on Two-dimensional Triangular Meshes}

In this subsection we give the reader an easy introduction to divergence-free reconstruction of the magnetic field within a triangular element. The easiest way is to focus on the triangle shown in Fig. 2 and consider just the second order case. For this very simple case, we will build up all the formulae explicitly. We also sketch out the third order case. Once those two cases are presented, the extension to all higher orders should be transparent. (Please note that because our method is based on classical zone-centered WENO reconstruction, which extends to all orders, our present method also extends to all orders. I.e. our method requires nothing but a classical finite volume WENO algorithm, coupled with the innovations reported here. This will become even more apparent in Section V.) In Section V we show how the divergence-free reconstruction procedure that is described here can be automated on a computer at all orders of accuracy. We should mention that a similar process is also described in Sub-section 3.2.6 of Xu 
et al. [73]; but the notation in that paper is not as transparent as the notation here. Also see Appendix A.5 of Xu et al. [73].

Assume, therefore, that the WENO reconstruction has produced a piecewise linear profile in eqn. (2.6). Equations that are analogous to eqn. (2.6) are also posited for the other two faces of Fig. 2. Assume too that such linear profiles are available at each face of the triangle in Fig. 2. As always, we consider the simple case where all the normal vectors in Fig. 2 are outward-pointing. Therefore, in segment $P_{1} P_{2}$ we have two pieces of information, $\bar{B}_{1}^{n}$ and $\left(\partial_{l 1} B_{1}^{n}\right)$. In segment $P_{2} P_{3}$ we have two pieces of information, $\bar{B}_{2}^{n}$ and $\left(\partial_{l 2} B_{2}^{n}\right)$. Similarly, in segment $P_{3} P_{1}$ we have two pieces of information, $\bar{B}_{3}^{n}$ and $\left(\partial_{l 3} B_{3}^{n}\right)$. These six pieces of information are not independent. Because of eqn. (2.4) we only have five independent pieces of information residing in the three faces of Fig. 2. Using this information, we wish to obtain a second order accurate divergencefree magnetic field everywhere within the triangular element shown in Fig. 2. Consider the most general specification of a piecewise linear magnetic field within the triangular element. It is given by

$$
B_{x}(x, y)=a_{0}+a_{x} x+a_{y} y \quad ; \quad B_{y}(x, y)=b_{0}+b_{x} x+b_{y} y
$$

Eqn. (4.1) has six coefficients. However, because of the local divergence-free condition on the high order reconstruction given by (3.1), only five of them are independent, as shown by the equation below

$$
\partial_{x} B_{x}(x, y)+\partial_{y} B_{y}(x, y)=0 \Rightarrow a_{x}+b_{y}=0
$$

Notice, therefore, that the five independent pieces of information in the boundary of Fig. 2 are matched exactly by the five independent coefficients in eqn. (4.1) which holds inside the triangle shown in Fig. 2. This will allow us to satisfy also the second condition on a divergence free piecewise polynomial reconstruction, given by relation (3.2). Our task is now to find five linear equations for the five coefficients in eqn. (4.1).

Putting eqn. (2.2) into eqn. (4.1) and projecting the magnetic field along the normal vector $\mathbf{n}_{1}$ gives us

$$
\begin{aligned}
& n_{1 x} B_{x}\left(x\left(l_{1}\right), y\left(l_{1}\right)\right)+n_{1 y} B_{y}\left(x\left(l_{1}\right), y\left(l_{1}\right)\right)= \\
& {\left[\left(n_{1 x} a_{0}+n_{1 y} b_{0}\right)+\left(n_{1 x} a_{x}+n_{1 y} b_{x}\right) x_{C 1}+\left(n_{1 x} a_{y}+n_{1 y} b_{y}\right) y_{C 1}\right] } \\
&+ {\left[-n_{1 y}\left(n_{1 x} a_{x}+n_{1 y} b_{x}\right)+n_{1 x}\left(n_{1 x} a_{y}+n_{1 y} b_{y}\right)\right] l_{1} }
\end{aligned}
$$

Now matching the constant part of eqn. (4.3) with the constant part in eqn. (2.6) gives us 


$$
\left(n_{1 x} a_{0}+n_{1 y} b_{0}\right)+\left(n_{1 x} a_{x}+n_{1 y} b_{x}\right) x_{C 1}+\left(n_{1 x} a_{y}+n_{1 y} b_{y}\right) y_{C 1}=\bar{B}_{1}^{n}
$$

Likewise, matching the linear part of eqn. (4.3) with the linear part in eqn. (2.6) gives us

$$
-n_{1 y}\left(n_{1 x} a_{x}+n_{1 y} b_{x}\right)+n_{1 x}\left(n_{1 x} a_{y}+n_{1 y} b_{y}\right)=\left(\partial_{l 1} B_{1}^{n}\right)
$$

The previous two equations were obtained by focusing on segment $P_{1} P_{2}$. Equations that are analogous to eqn. (2.2) are also posited for the other two faces of Fig. 2. Doing the same procedure for segment $P_{2} P_{3}$ gives

$$
\left(n_{2 x} a_{0}+n_{2 y} b_{0}\right)+\left(n_{2 x} a_{x}+n_{2 y} b_{x}\right) x_{C 2}+\left(n_{2 x} a_{y}+n_{2 y} b_{y}\right) y_{C 2}=\bar{B}_{2}^{n}
$$

and

$-n_{2 y}\left(n_{2 x} a_{x}+n_{2 y} b_{x}\right)+n_{2 x}\left(n_{2 x} a_{y}+n_{2 y} b_{y}\right)=\left(\partial_{l 2} B_{2}^{n}\right)$

Likewise, focusing on segment $P_{3} P_{1}$ gives

$$
\left(n_{3 x} a_{0}+n_{3 y} b_{0}\right)+\left(n_{3 x} a_{x}+n_{3 y} b_{x}\right) x_{C 3}+\left(n_{3 x} a_{y}+n_{3 y} b_{y}\right) y_{C 3}=\bar{B}_{3}^{n}
$$

and

$$
-n_{3 y}\left(n_{3 x} a_{x}+n_{3 y} b_{x}\right)+n_{3 x}\left(n_{3 x} a_{y}+n_{3 y} b_{y}\right)=\left(\partial_{l 3} B_{3}^{n}\right)
$$

Eqn. (2.4) shows us that the three equations, (4.4), (4.6) and (4.8), are not mutually independent. Consequently, only two equations out of the three equations, (4.4), (4.6) and (4.8), are used. All three equations, (4.5), (4.7) and (4.9) are used. This gives us the desired $5 \times 5$ system of equations for the coefficients in eqn. (4.1).

It is worth observing that all the coefficients on the left hand sides of eqns. (4.4) to (4.9) only depend on the geometry of the triangle. Only the right hand sides of eqns. (4.4) to (4.9) change as time evolves. This is consistent with the fact that the divergence-free condition is a topological constraint on the structure of the magnetic field. If the mesh does not move from one timestep to another, the $5 \times 5$ system of equations can be inverted once and for all within each zone. The inverse matrix can be stored, further speeding up the divergence-free reconstruction. Lastly, it is important to recall that we are now ready to overwrite $\mathbf{B}_{i}^{n}:=a_{0} \hat{\mathbf{x}}+b_{0} \hat{\mathbf{y}}$. This ensures that the zone-averaged magnetic field is actually connected to, and depends on, the facial magnetic field components. The zone-averaged magnetic field is truly just a helping variable that helps us retain order of accuracy in the algorithm. The detailed description of second order accurate divergence-free reconstruction on triangular elements is now complete. 
We now turn to a description of third order accurate divergence-free reconstruction. Assume, therefore, that the WENO reconstruction has produced a piecewise quadratic profile in eqn. (2.6). Equations that are analogous to eqn. (2.6) are also posited for the other two faces of Fig. 2. Assume too that such quadratic profiles are available at each face of the triangle in Fig. 2. As always, we consider the simple case where all the normal vectors in Fig. 2 are outwardpointing. Therefore, in segment $P_{1} P_{2}$ we have three pieces of information, $\bar{B}_{1}^{n},\left(\partial_{l 1} B_{1}^{n}\right)$ and $\left(\partial_{l 1}^{2} B_{1}^{n}\right)$. In segment $P_{2} P_{3}$ we have three pieces of information, $\bar{B}_{2}^{n},\left(\partial_{l 2} B_{2}^{n}\right)$ and $\left(\partial_{l 2}^{2} B_{2}^{n}\right)$. Similarly, in segment $P_{3} P_{1}$ we have three pieces of information, $\bar{B}_{3}^{n},\left(\partial_{l 3} B_{3}^{n}\right)$ and $\left(\partial_{l 3}^{2} B_{3}^{n}\right)$. These nine pieces of information are not independent. Because of eqn. (2.4), we only have eight independent pieces of information residing in the three faces of Fig. 2. Using this information, we wish to obtain a third order accurate divergence-free magnetic field everywhere within the triangular element shown in Fig. 2. Consider the most general specification of a piecewise quadratic magnetic field within the triangular element. It is given by

$$
\begin{aligned}
& B_{x}(x, y)=a_{0}+a_{x} x+a_{y} y+a_{x x} x^{2}+a_{x y} x y+a_{y y} y^{2} \\
& B_{y}(x, y)=b_{0}+b_{x} x+b_{y} y+b_{x x} x^{2}+b_{x y} x y+b_{y y} y^{2}
\end{aligned}
$$

Eqn. (4.10) has twelve coefficients. However, because of the local divergence-free constraint (3.1), only nine of them are independent as shown in the equation below

$$
\partial_{x} B_{x}(x, y)+\partial_{y} B_{y}(x, y)=0 \Rightarrow a_{x}+b_{y}=0 ; 2 a_{x x}+b_{x y}=0 ; a_{x y}+2 b_{y y}=0
$$

Notice, therefore, that the eight independent pieces of information in the boundary of Fig. 2 now have to be matched by the nine independent coefficients in eqn. (4.10) which holds inside the triangle shown in Fig. 2. A possible simplification would be to set $a_{y y}=b_{x x}$ in eqn. (4.10), thereby bringing the number of independent coefficients down to eight. (Notice that we pick the highest order terms in eqn. (4.10) that do not participate in the divergence-free constraint. That way, all the variation in the lower order terms is fully retained. I.e., we would like to minimize the variation in a least squares sense.) A divergence-free magnetic field just does not have these extra degrees of freedom where $a_{y y}$ and $b_{x x}$ can be set independently. Therefore, equating them does not damage the order property.

An equation that is analogous to eqn. (4.3) can be built. The coefficients in eqn. (4.10), along with the constraints in eqn. (4.11) can be adjusted so that the divergence-free magnetic field within the zone coincides with the piecewise quadratic variation in the boundary of Fig. 2. The solution procedure can be automated on a computer following the general description of the weak form of the constrained $\mathrm{L}_{2}$ projection given in Section $\mathrm{V}$. Once the divergence-free reconstruction has been built in a zone, we are now ready to overwrite $\mathbf{B}_{i}^{n}$. In the third order case, the zone-averaged magnetic field depends not only on $a_{0}$ and $b_{0}$ in eqn. (4.10). Instead, it also 
depends on the quadratic terms in eqn. (4.10). This completes our description of third order accurate divergence-free reconstruction in two-dimensions. Such a process can be extended to fourth and higher orders, see the next Section $\mathrm{V}$ for all details.

The observant reader will notice that the divergence-free reconstruction described here is completely linear, i.e. it is based on the inversion of $5 \times 5$ or $8 \times 8$ matrices. As a result, it is fair to ask about the non-linear hybridization that is required to stabilize the magnetic field. Notice that such a non-linear hybridization was built into the zone-centered classical WENO reconstruction in Section III. As a result, the linear or quadratic variations in eqn. (2.6) have already been nonlinearly stabilized. In this Sub-section we only show how to take those non-linearly stabilized variations that reside in the boundary of the triangular element and build a divergence-free magnetic field everywhere within the triangular element.

Within each face of the triangle we will want to invoke one-dimensional Riemann solvers. For that Riemann problem to be well-defined, the normal component of the magnetic field has to be continuous at all the interior faces of the mesh. Our construction in this Section is such that this requirement is ensured.

\section{IV.b) Divergence-free Reconstruction of the Magnetic Field on Three-dimensional Tetrahedral Meshes}

The easiest way to introduce the reader to divergence-free reconstruction within the tetrahedral element shown in Fig. 3 is to consider just the second order case. For this very simple case, we will build up all the formulae explicitly. We also sketch out the third order case. Once those two cases are presented, the extension to all higher orders is transparent. In Section V we show how the divergence-free reconstruction procedure that is described here can be automated on a computer at all orders of accuracy. While the two-dimensional case has been discussed before in $\mathrm{Xu}$ et al. [73], the present discussion is original to the best of our knowledge.

Assume, therefore, that the WENO reconstruction has produced a piecewise linear profile in eqn. (2.13). Equations that are analogous to eqn. (2.13) are also posited for the other three faces of Fig. 3. Assume too that such linear profiles are available at each face of the tetrahedron in Fig. 3. As always, we consider the simple case where all the normal vectors in Fig. 3 are outward-pointing. Therefore, in $\Delta P_{1} P_{2} P_{3}$ we have three pieces of information, $\bar{B}_{1}^{n},\left(\partial_{\xi} B_{1}^{n}\right)$ and $\left(\partial_{\psi} B_{1}^{n}\right)$. Likewise, in $\Delta P_{1} P_{3} P_{4}$ we have three pieces of information, $\bar{B}_{2}^{n},\left(\partial_{\xi} B_{2}^{n}\right)$ and $\left(\partial_{\psi} B_{2}^{n}\right)$. Similarly, in $\Delta P_{1} P_{4} P_{2}$ we have three pieces of information, $\bar{B}_{3}^{n},\left(\partial_{\xi} B_{3}^{n}\right)$ and $\left(\partial_{\psi} B_{3}^{n}\right)$. Furthermore, in $\Delta P_{4} P_{3} P_{2}$ we have three pieces of information, $\bar{B}_{4}^{n},\left(\partial_{\xi} B_{4}^{n}\right)$ and $\left(\partial_{\psi} B_{4}^{n}\right)$. These twelve pieces of information are not independent. Because of eqn. (2.10) we only have eleven independent pieces of information residing in the four faces of Fig. 3. Using this information, we wish to obtain a second order accurate divergence-free magnetic field everywhere within the 
tetrahedral element shown in Fig. 3. Consider the most general specification of a piecewise linear magnetic field within the tetrahedral element. It is given by

$$
\begin{aligned}
& B_{x}(x, y, z)=a_{0}+a_{x} x+a_{y} y+a_{z} z \\
& B_{y}(x, y, z)=b_{0}+b_{x} x+b_{y} y+b_{z} z \\
& B_{z}(x, y, z)=c_{0}+c_{x} x+c_{y} y+c_{z} z
\end{aligned}
$$

Eqn. (4.12) has twelve coefficients. However, because of the local divergence-free constraint (3.1), only eleven of them are independent as shown by the equation below

$$
\partial_{x} B_{x}(x, y, z)+\partial_{y} B_{y}(x, y, z)+\partial_{z} B_{z}(x, y, z)=0 \quad \Rightarrow \quad a_{x}+b_{y}+c_{z}=0
$$

Notice, therefore, that the eleven independent pieces of information in the boundary of Fig. 3 are matched exactly by the eleven independent coefficients in eqn. (4.12) which holds inside the tetrahedron shown in Fig. 3. Our task is now to find eleven linear equations for the eleven coefficients in eqn. (4.12) that also satisfy condition (3.2).

Putting eqn. (2.8) into eqn. (4.12) and projecting the magnetic field along the normal vector $\mathbf{n}_{1}$ gives us

$$
\begin{aligned}
& n_{1 x} B_{x}(x(\xi, \psi), y(\xi, \psi), z(\xi, \psi))+n_{1 y} B_{y}(x(\xi, \psi), y(\xi, \psi), z(\xi, \psi)) \\
& +n_{1 z} B_{z}(x(\xi, \psi), y(\xi, \psi), z(\xi, \psi))= \\
& {\left[\left(n_{1 x} a_{0}+n_{1 y} b_{0}+n_{1 z} c_{0}\right)+\left(n_{1 x} a_{x}+n_{1 y} b_{x}+n_{1 z} c_{x}\right) x_{C 1}+\left(n_{1 x} a_{y}+n_{1 y} b_{y}+n_{1 z} c_{y}\right) y_{C 1}+\left(n_{1 x} a_{z}+n_{1 y} b_{z}+n_{1 z} c_{z}\right) z_{C 1}\right]} \\
& +\left[\xi_{1 x}\left(n_{1 x} a_{x}+n_{1 y} b_{x}+n_{1 z} c_{x}\right)+\xi_{1 y}\left(n_{1 x} a_{y}+n_{1 y} b_{y}+n_{1 z} c_{y}\right)+\xi_{1 z}\left(n_{1 x} a_{z}+n_{1 y} b_{z}+n_{1 z} c_{z}\right)\right] \xi \\
& +\left[\psi_{1 x}\left(n_{1 x} a_{x}+n_{1 y} b_{x}+n_{1 z} c_{x}\right)+\psi_{1 y}\left(n_{1 x} a_{y}+n_{1 y} b_{y}+n_{1 z} c_{y}\right)+\psi_{1 z}\left(n_{1 x} a_{z}+n_{1 y} b_{z}+n_{1 z} c_{z}\right)\right] \psi
\end{aligned}
$$

Now matching the constant part of eqn. (4.14) with the constant part in eqn. (2.13) gives us

$$
\begin{aligned}
& \left(n_{1 x} a_{0}+n_{1 y} b_{0}+n_{1 z} c_{0}\right)+\left(n_{1 x} a_{x}+n_{1 y} b_{x}+n_{1 z} c_{x}\right) x_{C 1} \\
& +\left(n_{1 x} a_{y}+n_{1 y} b_{y}+n_{1 z} c_{y}\right) y_{C 1}+\left(n_{1 x} a_{z}+n_{1 y} b_{z}+n_{1 z} c_{z}\right) z_{C 1}=\bar{B}_{1}^{n}
\end{aligned}
$$

Likewise, matching the linear parts of eqn. (4.14) with the linear parts in eqn. (2.13) gives us

$$
\xi_{1 x}\left(n_{1 x} a_{x}+n_{1 y} b_{x}+n_{1 z} c_{x}\right)+\xi_{1 y}\left(n_{1 x} a_{y}+n_{1 y} b_{y}+n_{1 z} c_{y}\right)+\xi_{1 z}\left(n_{1 x} a_{z}+n_{1 y} b_{z}+n_{1 z} c_{z}\right)=\left(\partial_{\xi} B_{1}^{n}\right)
$$

and

$$
\psi_{1 x}\left(n_{1 x} a_{x}+n_{1 y} b_{x}+n_{1 z} c_{x}\right)+\psi_{1 y}\left(n_{1 x} a_{y}+n_{1 y} b_{y}+n_{1 z} c_{y}\right)+\psi_{1 z}\left(n_{1 x} a_{z}+n_{1 y} b_{z}+n_{1 z} c_{z}\right)=\left(\partial_{\psi} B_{1}^{n}\right)
$$


Eqns. (4.15) to (4.17) represent three equations that we get from $\Delta P_{1} P_{2} P_{3}$, i.e. the first face of the tetrahedral element shown in Fig. 3. There are three more faces. By replacing the subscript "1" in eqns. (4.15) to (4.17) with the subscript " 2 ", we get three more equations that hold in the second face of the tetrahedral element shown in Fig. 3. To get three more equations in the third face of the tetrahedral element shown in Fig. 3, replace the subscript "1" in eqns. (4.15) to (4.17) with the subscript " 3 ". Likewise, to get three more equations in the fourth face of the tetrahedral element shown in Fig. 3, replace the subscript "1" in eqns. (4.15) to (4.17) with the subscript "4".

In each of the four faces of the tetrahedron, we have eqn. (4.15) or one of its variants. Because of eqn. (2.10), those four equations are not mutually independent. Consequently, we can only pick three of them. However, we can pick all four variants of eqn. (4.16) and all four variants of eqn. (4.17). The result is an $11 \times 11$ system of equations for the eleven independent coefficients of eqn. (4.13). When the mesh does not move from one timestep to another, it is even possible to invert the $11 \times 11$ system of equations within each zone and store the inverse. Lastly, it is important to recall that we are now ready to overwrite $\mathbf{B}_{i}^{n}:=a_{0} \hat{\mathbf{x}}+b_{0} \hat{\mathbf{y}}+c_{0} \hat{\mathbf{z}}$. This ensures that the zone-averaged magnetic field is actually connected to, and depends on, the facial magnetic field components. The detailed description of second order accurate divergence-free reconstruction on tetrahedral elements is now complete.

We now turn our attention to a description of third order accurate divergence-free reconstruction. Assume, therefore, that the WENO reconstruction has produced a piecewise quadratic profile in eqn. (2.13). Equations that are analogous to eqn. (2.13) are also posited for the other three faces of Fig. 3. Assume too that such quadratic profiles are available at each face of the tetrahedron in Fig. 3. As always, we consider the simple case where all the normal vectors in Fig. 3 are outward-pointing. Therefore, in $\Delta P_{1} P_{2} P_{3}$ we have six pieces of information, $\bar{B}_{1}^{n}$, $\left(\partial_{\xi} B_{1}^{n}\right),\left(\partial_{\psi} B_{1}^{n}\right),\left(\partial_{\xi \xi}^{2} B_{1}^{n}\right),\left(\partial_{\xi \psi}^{2} B_{1}^{n}\right)$ and $\left(\partial_{\psi \psi}^{2} B_{1}^{n}\right)$. The other three faces of the tetrahedron will also contain six pieces of information each. Because of eqn. (2.10) we only have twenty-three independent pieces of information residing in the four faces of Fig. 3. Using this information, we wish to obtain a third order accurate divergence-free magnetic field everywhere within the tetrahedral element shown in Fig. 3. Consider the most general specification of a piecewise quadratic magnetic field within the tetrahedral element. It is given by

$$
\begin{aligned}
& B_{x}(x, y, z)=a_{0}+a_{x} x+a_{y} y+a_{z} z+a_{x x} x^{2}+a_{y y} y^{2}+a_{z z} z^{2}+a_{x y} x y+a_{y z} y z+a_{x z} x z ; \\
& B_{y}(x, y, z)=b_{0}+b_{x} x+b_{y} y+b_{z} z+b_{x x} x^{2}+b_{y y} y^{2}+b_{z z} z^{2}+b_{x y} x y+b_{y z} y z+b_{x z} x z ; \\
& B_{z}(x, y, z)=c_{0}+c_{x} x+c_{y} y+c_{z} z+c_{x x} x^{2}+c_{y y} y^{2}+c_{z z} z^{2}+c_{x y} x y+c_{y z} y z+c_{x z} x z
\end{aligned}
$$

Eqn. (4.18) has thirty coefficients. However, because of the local divergence-free constraint given by relation (3.1), only twenty-six of them are independent, as shown by the equation below 


$$
\begin{aligned}
& \partial_{x} B_{x}(x, y, z)+\partial_{y} B_{y}(x, y, z)+\partial_{z} B_{z}(x, y, z)=0 \Rightarrow a_{x}+b_{y}+c_{z}=0 ; 2 a_{x x}+b_{x y}+c_{x z}=0 ; \\
& a_{x y}+2 b_{y y}+c_{y z}=0 ; a_{x z}+b_{y z}+2 c_{z z}=0
\end{aligned}
$$

In principle, one might use least squares minimization in this situation. However, as with the third order case in two-dimensions, it is possible to restrict the variation in terms at the highest order so that all the essential variation in the lower order terms is exactly preserved. Several options are available for reducing the number of independent coefficients in eqn. (4.18). We can try the first option

$$
a_{y z}=0 ; b_{x z}=0 ; c_{x y}=0
$$

or we can try the second option

$$
b_{x x}=c_{x x} \quad ; \quad a_{y y}=c_{y y} \quad ; \quad a_{z z}=b_{z z}
$$

or the third option

$$
a_{y y}=a_{z z} ; b_{x x}=b_{z z} \quad ; \quad c_{x x}=c_{y y}
$$

An equation that is analogous to eqn. (4.14) can be built and used to match to the piecewise quadratic variation in the boundary of Fig. 3. The solution procedure can be automated on a computer. Once the divergence-free reconstruction has been built in a zone, we are now ready to overwrite $\mathbf{B}_{i}^{n}$. In the third order case, the zone-averaged magnetic field is not only dependent on $a_{0}, b_{0}$ and $c_{0}$ in eqn. (4.18). Instead, it also depends on the quadratic terms in eqn. (4.18). This completes our description of third order accurate divergence-free reconstruction in threedimensions. Such a process can be extended to fourth and higher orders, see the next Section V for all details.

\section{V) Divergence-free Reconstruction of the Magnetic Field Based on Constrained $L_{2}$ Projection}

The motivation for the narrative in Section IV was to show that one can start with the face-averaged normal components and a classical zone-based WENO reconstruction of the magnetic field in order to arrive at a divergence-free reconstruction of the magnetic field within each zone that satisfies conditions (3.1) and (3.2). We showed that this can, in principle, be done at all orders. Its computer implementation at each order would, however, be very cumbersome.

In the following section we will present a new and general strategy for producing such a high order accurate divergence-free reconstruction of the magnetic field. As input, the new method requires a classical high order accurate, but non divergence-free, zone centered WENO reconstruction of the magnetic field. It further requires as input a set of face-averaged magnetic 
field components that are divergence-free in the sense of eqn. (2.4) and (2.10). On output, the new method returns a high order zone-centered polynomial reconstruction of the magnetic field that is locally and globally divergence-free, i.e. that satisfies (3.1) and (3.2). In Sub-Section V.a we first motivate the need for our new constrained $\mathrm{L}_{2}$ projection-based scheme and then in Subsection V.b we present all the necessary details.

\section{V.a) Motivation for a more general approach - comparing the number of constraints with the number of degrees of freedom for the magnetic field}

The case studies in Section IV have shown that the second order divergence-free reconstructions in two- and three-dimensions have a special property, namely that the number of unknowns in the reconstructed polynomial exactly match the number of constraints provided by the face-averaged magnetic field components and their first moments on the element boundary. In general, Section IV also shows us that for third and higher order schemes on simplex elements the number of unknown degrees of freedom for the divergence-free magnetic field is in general larger than the number of available constraints on the element boundary and within each element. Therefore, it became necessary to choose additional ad hoc equations based on symmetry considerations, such as e.g. (4.20), in order to close the system. In Section IV we had an inkling that a least squares-like approach might provide a resolution for this problem. In this motivational sub-section we show that the use of a constrained least squares type approach is inevitable for very high order schemes. In the next sub-section we show how such a process can be automated on a computer.

In the following we denote the number of degrees of freedom associated with a polynomial of maximum degree $N$ in $d$ space dimensions with the symbol $\mathrm{N}=\mathrm{N}(N, d)$, given by the relation

$\mathrm{N}(N, d)=\frac{\prod_{i=1}^{d}(N+i)}{d !}$

For example, each of the degree one polynomials in the two-dimensional eqn. (4.1) has $\mathrm{N}(1,2)=3$, while each of the degree one polynomials in the three-dimensional eqn. (4.12) has $\mathrm{N}(1,3)=4$. Likewise, each of the degree two polynomials in the two-dimensional eqn. (4.10) has $N(2,2)=6$, while each of the degree two polynomials in the three-dimensional eqn. (4.18) has $\mathrm{N}(2,3)=10$.

The number of unknowns for the divergence-free magnetic field is then $d \cdot \mathrm{N}(N, d)$, since each component of the magnetic field vector is reconstructed by a polynomial of degree $N$. The number of constraints to get a locally divergence-free magnetic field within each zone is $\mathrm{N}(N-1, d)$. Matching the face-averaged magnetic fields adds additional $n_{f}-1$ constraints, 
where $n_{f}$ denotes the number of faces for each element (i.e., $n_{f}=3$ for triangles and $n_{f}=4$ for tetrahedra). Recall from eqns. (2.4) and (2.10) that one of the magnetic field components in one of the faces of the element is redundant because of the divergence-free property. The remaining number of constraints for the higher order moments on the faces of an element is equal to $n_{f} \cdot(\mathrm{N}(N, d-1)-1)$. By putting it all together, we obtain the number of additional equations, $n_{A}$, that must be specified in order to determine a higher order piecewise polynomial divergencefree magnetic field according to relations (3.1) and (3.2). It is obtained by taking the difference between the number of unknowns and the number of constraints:

$n_{A}=d \cdot \mathrm{N}(N, d)-\mathrm{N}(N-1, d)-\left(n_{f}-1\right)-n_{f} \cdot(\mathrm{N}(N, d-1)-1)$

We examine this equation in detail in the next paragraph.

For triangles, with $d=2$, the number of additional ( $\mathrm{ad}$ hoc) equations that need to be specified in order to define a divergence-free magnetic field is

$n_{A}^{\text {tri }}=\frac{1}{2} N(N-1)$

For tetrahedra, with $d=3$, the number of additional ( $\mathrm{ad}$ hoc) equations that need to be specified in order to define a divergence-free magnetic field is

$n_{A}^{\text {tetra }}=\frac{1}{6} N(2 N+5)(N-1)$

It is obvious from eqns. (5.3) and (5.4) that for second order schemes, i.e. with $N=1$, the number of unknowns is equal to the number of constraints. However, for third and higher order schemes, i.e. with $N>1$, the number $n_{A}$ grows quickly with increasing polynomial degree, in particular for the three dimensional case. We therefore realize that it is not enough to require only that the divergence-free polynomial reconstruction within a zone satisfies the constraints (3.1) and (3.2). Instead, we need an additional ingredient for its construction. Such a new ingredient is proposed in this paper in the following: we will require that the divergence-free polynomial to be found is in some sense also "as close as possible" to the original zone-centered WENO reconstruction of the magnetic field, while satisfying at the same time also the constraints (3.1) and (3.2). The new approach for obtaining a high order divergence-free magnetic field inside each zone is based on a novel constrained $\mathrm{L}_{2}$ projection that is described in detail below.

\section{V.b) Constrained $\mathrm{L}_{2}$ projection}

At the beginning of each timestep we have our zone-averaged flow variables of density, momentum and energy density from eqn. (1.2). We also have the face-averaged magnetic field components, which are indeed divergence-free according to (2.4) and (2.10). We also have an 
auxiliary zone-averaged magnetic field that has been obtained from a previous timestep. However, since the previous timestep was order preserving in all the zone-averaged fluid and magnetic variables, the zone-averaged magnetic field will be accurate enough to be used for the reconstruction process. Our overarching goal is to simplify the reconstruction by basing it on the well-known classical zone-based WENO reconstruction algorithm from Dumbser \& Käser [39]. This reconstruction strategy acts on all the zone-averaged variables, including the zone-averaged magnetic field vector. The zone-averaged magnetic field is, therefore, an auxiliary variable that helps us with the initial WENO reconstruction.

Within each element $T_{i}$ let us define the preliminary reconstruction polynomial of the magnetic field vector that is the result of a classical WENO reconstruction according to Dumbser $\&$ Käser [39]. Recall that this classical WENO reconstruction at time $t^{n}$ does in general not yet satisfy the local and global divergence-free condition (3.1) and (3.2). It is written as

$$
\mathbf{B}_{h}^{n}\left(\mathbf{x}, t^{n}\right)=\sum_{m} \psi_{m}(\mathbf{x}) \hat{\mathbf{B}}_{i, m}^{n}:=\psi_{m}(\mathbf{x}) \hat{\mathbf{B}}_{i, m}^{n}, \quad \forall \mathbf{x} \in T_{i}
$$

where we have used the Einstein summation convention over two repeated indices. In the relation above, the functions $\psi_{m} \in \mathrm{P}_{N}^{d}$ are the orthogonal reconstruction basis functions from the space $\mathrm{P}_{N}^{d}$ of piecewise polynomials of degree $N$ in $d$ space dimensions. The $\hat{\mathbf{B}}_{m}^{n}$ are the so-called degrees of freedom, or moments, and are obtained from the classical Dumbser \& Käser WENO reconstruction [39]. For example, at second order, eqn. (5.5) will have as many terms as are contained in eqns. (4.1) and (4.12) in two- and three-dimensions respectively. Likewise, at third order, eqn. (5.5) will have as many terms as are contained in eqns. (4.10) and (4.18) in two- and three-dimensions respectively. However, the magnetic field defined by eqn. (5.5) is in general not yet divergence-free, because it does not satisfy the constraints given in eqns. (3.1) and (3.2). The task of the constrained $\mathrm{L}_{2}$ projection is now to make a small modification to the degrees of freedom in eqn. (5.5) that are in some sense optimal. The modified version of eqn. (5.5) should be divergence-free within the element and must match the moments of the facial magnetic field components, according to (3.1) and (3.2).

Let $\xi$ be a " $d-1$ " dimensional coordinate system within the face $F_{j}$. Note that

$$
B_{j}\left(\xi, t^{n}\right)=\Phi_{m}(\xi) \widehat{B}_{j, m}^{n}
$$

is our short-hand notation for a high order polynomial expansion of the normal component of the magnetic field inside each oriented face $F_{j}$ in terms of some orthogonal basis functions $\Phi_{m}(\xi) \in \mathrm{P}_{N}^{d-1}$. I.e., the basis functions $\Phi_{m}(\xi)$ are again piecewise polynomials of degree $N$, but in one spatial dimension less than the basis functions $\psi_{m}$. For example, notice that eqns. (2.6) and (2.13) are written in terms of facial coordinates in two- and three-dimensions respectively. 
The mean value of the magnetic field component and its derivatives within eqns. (2.6) and (2.13) are indeed the facial modes, $\widehat{B}_{j, m}^{n}$, in eqn. (5.6).

In this paper we propose to use classical one-dimensional Legendre polynomials for the faces of triangular grids and the two-dimensional Dubiner basis on triangles in case of a threedimensional tetrahedral mesh. The zeroth order moment of this expansion within each face is set to the face averaged normal magnetic field component defined within $F_{j}$, i.e. we set $\widehat{B}_{j, 0}^{n}:=\bar{B}_{j}^{n}$. The higher order moments for $j>0$ are obtained from the preliminary WENO reconstruction polynomials $\mathbf{B}_{h}\left(\mathbf{x}, t^{n}\right)$ using $\mathrm{L}_{2}$ projection and a minmod technique as follows:

$\widehat{B}_{j, m}^{n}=\operatorname{minmod}\left(\widehat{B}_{j, m}^{-}, \widehat{B}_{j, m}^{+}\right), \quad \forall m>0$

with

$$
\int_{F_{j}} \Phi_{m}(\xi) \widehat{B}_{j, m}^{ \pm} d \xi=\int_{F_{j}} \Phi_{m}(\boldsymbol{\xi}) \mathbf{B}_{h}^{n}\left(\xi^{ \pm}, t^{n}\right) \cdot \mathbf{n}_{j} d \xi, \quad \forall m>0
$$

where the $\xi^{ \pm}$denotes the left and the right side of the oriented face $F_{j}$, respectively. Notice that the mean values of the magnetic field components are left unchanged. However, the higher moments in each face can be obtained from our classical WENO reconstruction from either side of the face. It is by this simplification that we avoid the complicated facial WENO reconstruction from Xu et al. [73].

At time $t^{n}$ we are now looking for a divergence-free reconstruction within the element $T_{i}$ of the magnetic field that satisfies (3.1) and (3.2) and which is denoted by

$\tilde{\mathbf{B}}_{h}^{n}\left(\mathbf{x}, t^{n}\right)=\Psi_{m}(\mathbf{x}) \tilde{\mathbf{B}}_{i, m}^{n}$,

It should be as close as possible to the original WENO polynomial $\mathbf{B}_{h}^{n}\left(\mathbf{x}, t^{n}\right)$ from eqn. (5.5). We bring about this proximity in the $\mathrm{L}_{2}$ norm because this is a physically-motivated energy norm for the magnetic field, as will be explained later. A least squares approach is a natural way to minimize this distance. But recall from our study of Section IV that divergence-free reconstruction also requires the satisfaction of constraints. Consequently, we need to use a constrained optimization method that minimizes the distance in $\mathrm{L}_{2}$ norm, while satisfying the constraints (3.1) and (3.2). In particular, the divergence-free polynomial has to satisfy:

i) We want the magnetic field to be locally divergence-free at each and every point inside all elements $T_{i}$, according to (3.1).

ii) We further require that for each face $F_{j} \in \mathrm{F}_{i}$ of the element, the normal component of the reconstructed polynomial of the magnetic field vector matches the higher order representation 
given by $B_{j}\left(\xi, t^{n}\right)$ pointwise according to (3.2). In order words, the normal component of the magnetic field must be pointwise continuous across each face $F_{j} \in \mathrm{F}_{i}$. This is achieved by requiring that our reconstructed polynomial matches the facially-averaged magnetic field component in each face of $T_{i}$. We also require that our reconstructed polynomial matches all the higher order moments of the normal component of the magnetic field in each face of $T_{i}$.

In a weak integral formulation this constrained $\mathrm{L}_{2}$ projection reads:

minimize : $\int_{T_{i}}\left(\tilde{\mathbf{B}}_{h}^{n}\left(\mathbf{x}, t^{n}\right)-\mathbf{B}_{h}^{n}\left(\mathbf{x}, t^{n}\right)\right)^{2} d \mathbf{x}$

with the three constraints :

$\int_{T_{i}} \Psi_{k}^{\prime} \nabla \cdot \tilde{\mathbf{B}}_{h}^{n}\left(\mathbf{x}, t^{n}\right) d \mathbf{x}=0, \quad \forall \Psi_{k}^{\prime} \in \mathrm{P}_{N-1}^{d}$,

$\int_{F_{j} \in \mathrm{F}_{i}^{\subset}} \tilde{\mathbf{B}}_{h}^{n}\left(\mathbf{x}, t^{n}\right) \cdot \mathbf{n}_{i j} d S=\int_{F_{j}} \sigma_{i j} \bar{B}_{j} d S, \quad \forall F_{j} \in \mathrm{F}_{i}^{\subset} \subset \mathrm{F}_{i}$.

$\int_{F_{j} \in \mathrm{F}_{i}} \Phi_{k} \tilde{\mathbf{B}}_{h}^{n}\left(\mathbf{x}, t^{n}\right) \cdot \mathbf{n}_{i j} d S=\int_{F_{j}} \Phi_{k} \sigma_{i j} B_{j}\left(\xi, t^{n}\right) d S, \quad \forall \Phi_{k} \in \mathrm{P}_{N}^{d-1} \backslash \mathrm{P}_{0}^{d-1}, \quad \forall F_{j} \in \mathrm{F}_{i}$.

Here the symbol $\mathrm{F}_{i}^{\subset} \subset \mathrm{F}_{i}$ denotes an arbitrary subset of $d$ faces from the $d+1$ faces contained in $\mathrm{F}_{i}$. Excluding one face from the set of faces $\mathrm{F}_{i}$ is necessary due to the fact that if the magnetic field is locally divergence-free (second relation in 5.10), the boundary integral is automatically divergence-free because of the Gauss theorem, hence using all faces $\mathrm{F}_{i}$ would give redundant information. The symbol $\Psi_{k}^{\prime}$ in (5.10) denotes test functions that are from the space of piecewise polynomials of degree $N-1$ in $d$ dimensions.

It is important to develop an intuitive feeling for the meaning of eqn. (5.10), which is discussed in the following. The first equation in (5.10) is the minimization of the distance between the auxiliary WENO reconstruction and the sought divergence-free reconstruction of the magnetic field in $\mathrm{L}_{2}$ norm. The second one enforces the divergence-free constraint locally and pointwise inside each element, hence imposing condition (3.1). The third one requires that the facial average $\bar{B}_{j}^{n}$ of the normal component of the magnetic field is matched exactly by the reconstruction. The fourth equation ensures that all the higher order moments of the normal component of the magnetic field are matched exactly on the face. The third and fourth equation of (5.10) are necessary to impose constraint (3.2) of our divergence-free reconstruction.

The first relation of (5.10) can equivalently be rewritten as min imize : $\int_{T_{i}}\left(\tilde{\mathbf{B}}_{h}^{n}\left(\mathbf{x}, t^{n}\right)-\mathbf{B}_{h}^{n}\left(\mathbf{x}, t^{n}\right)\right)^{2} d \mathbf{x}=\int_{T_{i}} \Psi_{k}(\mathbf{x}) \Psi_{m}(\mathbf{x})\left(\tilde{\mathbf{B}}_{i, k}^{n}-\hat{\mathbf{B}}_{i, k}^{n}\right)\left(\tilde{\mathbf{B}}_{i, m}^{n}-\hat{\mathbf{B}}_{i, m}^{n}\right) d \mathbf{x}$. 
The constraints in eqn. (5.10) are then added to the system using a classical Lagrangian multiplier technique [39], so the function $g$ to be minimized reads

$$
\begin{aligned}
& g=\int_{T_{i}}\left(\tilde{\mathbf{B}}_{h}^{n}\left(\mathbf{x}, t^{n}\right)-\mathbf{B}_{h}^{n}\left(\mathbf{x}, t^{n}\right)\right)^{2} d \mathbf{x}-\lambda\left(\int_{T_{i}} \Psi_{k}^{\prime} \nabla \cdot \tilde{\mathbf{B}}_{h}^{n}\left(\mathbf{x}, t^{n}\right) d \mathbf{x}\right)-\boldsymbol{\mu}\left(\int_{F_{j} \in \mathrm{F}_{i}^{c}}\left(\tilde{\mathbf{B}}_{h}^{n}\left(\mathbf{x}, t^{n}\right) \cdot \mathbf{n}_{i j}-\sigma_{i j} \bar{B}_{j}^{n}\right) d S\right) \\
& -\mathbf{v}\left(\int_{F_{j} \in \mathrm{F}_{i}} \Phi_{k}\left(\tilde{\mathbf{B}}_{h}^{n}\left(\mathbf{x}, t^{n}\right) \cdot \mathbf{n}_{i j}-\sigma_{i j} B_{j}\left(\xi, t^{n}\right)\right) d S\right) .
\end{aligned}
$$

In the above equation, the zonal degrees of freedom $\hat{\mathbf{B}}_{i, m}^{n}$ are known from our preliminary WENO reconstruction in eqn. (5.5). The facially-averaged magnetic field components, $\bar{B}_{j}^{n}$, are also known in the previous equation. Also, in the above equation, the facial degrees of freedom $\widehat{B}_{j, m}^{n}$ are known from eqns. (5.6) and (5.7). The divergence-free degrees of freedom $\tilde{\mathbf{B}}_{i, k}^{n}$ in eqn. (5.9) are indeed the unknowns that we wish to compute. They are obtained from eqn. (5.11) by enforcing the following conditions for our constrained optimization problem:

$\frac{\partial g}{\partial \widetilde{\mathbf{B}}_{i, m}^{n}}=0, \quad \frac{\partial g}{\partial \lambda}=0, \quad \frac{\partial g}{\partial \boldsymbol{\mu}}=0, \quad \frac{\partial g}{\partial \boldsymbol{v}}=0$.

I.e., we obtain the following linear equation system for the Lagrange multipliers $\boldsymbol{\lambda}, \boldsymbol{\mu}$ and $\boldsymbol{v}$ and also for the unknown degrees of freedom $\tilde{\mathbf{B}}_{i, k}^{n}$ of the final divergence-free magnetic field:

$$
\begin{aligned}
& \frac{\partial g}{\partial \tilde{\mathbf{B}}_{i, m}^{n}}=\left(2 \int_{T_{i}} \Psi_{k} \Psi_{m} d \mathbf{x}\right)\left(\tilde{\mathbf{B}}_{i, k}^{n}-\hat{\mathbf{B}}_{i, k}^{n}\right)-\lambda\left(\int_{T_{i}} \Psi_{k}^{\prime} \nabla \Psi_{m} d \mathbf{x}\right)-\boldsymbol{\mu}\left(\int_{F_{j} \in \mathrm{F}_{i}^{c}} \Psi_{m} \mathbf{n}_{i j} d S\right)-\mathbf{v}\left(\int_{F_{j} \in \mathrm{F}_{i}} \Phi_{k} \Psi_{m} \mathbf{n}_{i j} d S\right)=0, \\
& \frac{\partial g}{\partial \lambda}=\left(\int_{T_{i}} \Psi_{k}^{\prime} \nabla \Psi_{m} d \mathbf{x}\right) \cdot \tilde{\mathbf{B}}_{i, m}^{n}=0, \\
& \frac{\partial g}{\partial \boldsymbol{\mu}}=\int_{F_{j} \in \mathrm{F}_{i}^{\subset}}\left(\Psi_{m} \tilde{\mathbf{B}}_{i, m}^{n} \cdot \mathbf{n}_{i j}-\sigma_{i j} \bar{B}_{j}^{n}\right) d S=0, \\
& \frac{\partial g}{\partial \mathbf{v}}=\int_{F_{j} \in \mathrm{F}_{i}} \Phi_{k}\left(\Psi_{m} \tilde{\mathbf{B}}_{i, m}^{n} \cdot \mathbf{n}_{i j}-\sigma_{i j} \Phi_{m}(\boldsymbol{\xi}) \hat{B}_{j, m}^{n}\right) d S=0 .
\end{aligned}
$$

The resulting polynomial is actually a minimizer of the quadratic optimization problem because the mass matrix in (5.13) is symmetric and positive definite. In practice, the integrals in eqn. (5.13) are computed by using sufficiently high order numerical quadrature formulae enforced on the reference triangle or tetrahedron in two- and three-dimensions respectively. For polynomial bases, the quadrature formulae can be made exact, see the well-known book of Stroud [76]. As a result, the reconstructed magnetic field is pointwise divergence-free while matching the faceaveraged magnetic field components and their higher order moments at the zone faces, according to (3.1) and (3.2). This completes the general description of the constrained $\mathrm{L}_{2}$ projection algorithm. We stress that our constrained optimization approach is not minimizing the 
divergence of the magnetic field. In fact, after this optimization process, the divergence of the magnetic field is always exactly zero in the sense of (3.1) and (3.2). Instead, our method minimizes the distance in $\mathrm{L}_{2}$ norm of the sought after divergence-free polynomial, given by eqn. (5.9), to the preliminary WENO reconstruction polynomial, given by eqn. (5.5).

It is also worthwhile to note that in MHD, as in electromagnetism, the magnetic energy is given by the square of the magnetic field. As a result, our constrained optimization procedure minimizes the variance in the magnetic energy from the classical WENO reconstruction, consistent with preserving all the constraints. In Section IV, at third order, we were forced to make some ad hoc choices for some of the coefficients. See the paragraph after eqn. (4.11) for the two-dimensional case; or see eqn. (4.20) in the three-dimensional case. Our constrained $\mathrm{L}_{2}$ projection algorithm gets rid of such ambiguity, yielding a solution that is well-defined in an energy norm.

Once we have obtained the divergence-free magnetic field $\tilde{\mathbf{B}}_{h}^{n}\left(\mathbf{x}, t^{n}\right)$, given by eqn. (5.9), inside each zone from the solution of (5.13), we overwrite the auxiliary cell-average for the magnetic field $\mathbf{B}$ by the average of the divergence-free magnetic field as follows:

$\mathbf{B}_{i}^{n}:=\frac{1}{\left|T_{i}\right|} \int_{T_{i}} \tilde{\mathbf{B}}_{h}^{n}\left(\mathbf{x}, t^{n}\right) d \mathbf{x}$

Realize that eqn. (5.14) completes the discussion in this Section because it shows us how to build the true cell averaged magnetic field that only depends on the face-averaged magnetic field components and the high order divergence-free reconstruction. In the course of the timestep that takes us from time $t^{n}$ to time $t^{n+1}=t^{n}+\Delta t$, we will now evolve the facial magnetic field components using either eqn. (2.5) or eqn. (2.11) in two or three dimensions. We will also evolve the zone-averaged fluid variables, as well as the zone-averaged magnetic field variables from eqn. (5.14). This zone-averaged update is made by using a flux conservative approach from eqn. (1.2). The resulting auxiliary zone-averaged magnetic field at time $t^{n+1}$ will not be divergencefree; although it will have the desired order of accuracy. However, the face-averaged magnetic field components at time $t^{n+1}$ will indeed be divergence-free in the sense of (2.4) and (2.10) and they will also have the desired order of accuracy. At time $t^{n+1}$, the zone-averaged magnetic field will, nevertheless, be useful as an auxiliary variable because it will simplify the reconstruction process in the next timestep.

\section{VI) Pointwise Synopsis of the Algorithm}

In the following, we summarize all the necessary steps of our new algorithm in their proper sequential order:

1) At the start of the computation, initialize the zone-centered mass, momentum, energy densities and magnetic fields as well as the facial components of the divergence-free magnetic field. If an analytical divergence-free specification of the magnetic field vector is available for use in eqn. 
(2.3) then this can be done via exact integration. In practice, this is usually done by realizing that the magnetic field can be specified in terms of a magnetic vector potential $(\mathbf{B}=-\nabla \times \mathbf{A})$. Please see Balsara [8] for examples of a magnetic vector potential and how it is used to initialize a divergence-free magnetic field on a mesh.

2) At the start of each timestep, start with the conservative variables at the zone center. Then, compute the primitive variables at the zone center and use them to build eigenvalues and eigenvectors as needed. Since MHD is a non-convex system, reconstruction via characteristic variables proves to be particularly useful. Use the zone-centered reconstruction strategy from Dumbser \& Käser [39] to reconstruct all the flow variables including the magnetic fields. If needed, use the positivity-preserving strategy from Balsara [20]. Please also see Cheng, Li, Qiu $\& \mathrm{Xu}$ [26]. This completes the preliminary WENO reconstruction step.

3) Using the description from Section V, obtain a unique specification of the moments of the magnetic field components within the faces of each of the elements, see eqn. (5.7) and (5.8). Use the constrained $\mathrm{L}_{2}$ projection algorithm of Section $\mathrm{V}$ to make a divergence-free reconstruction of the magnetic field within the zone. Once the divergence-free reconstruction is in hand, use it to overwrite the auxiliary zone-averaged magnetic field at the aid of eqn. (5.14).

4) By the end of the previous step, the spatially reconstructed flow variables and the divergencefree, spatially reconstructed magnetic field variables are available within each zone. This is exactly the information that the PDE needs to evolve the system further in time. Use the ADER time-update strategy (Titarev \& Toro [70], [71], Toro \& Titarev [72], Dumbser et al. [36], [37], Balsara et al. [13], [14]) to obtain the space-time evolution of the MHD system within each zone.

5) Invoke the MuSIC Riemann solver at each edge, as described in the narrative surrounding eqn. (2.5). This should provide edge-centered electric field components and also multidimensional contributions to the face-centered fluxes. Using face-centered invocations of the one-dimensional Riemann solver, build a high order facial flux. As needed, use quadrature in time to build space-time accurate electric field components at the edges of the mesh and spacetime accurate fluxes at the faces of the mesh.

6) Use eqn. (2.5) to update the facial components of the magnetic field. The zone-centered mass, momentum and energy densities as well as the auxiliary zone-centered magnetic fields are updated in the usual conservative way using eqn. (1.2). This completes our description of a timestep of our MHD algorithm.

\section{VII) Accuracy Analysis}

We present two accuracy analyses, one in two-dimensions and the other in threedimensions. The magnetized isodensity vortex problem described in Balsara [8] consists of a magnetized vortex moving across a domain given by $[-5,5]^{2}$ in two-dimensions and by $[-5,5]^{3}$ in three-dimensions. The length of the domain along each dimension is denoted by $\mathrm{L}=10$ in the 
following. The vortex propagates in the $x-y$ plane at an angle of $45^{\circ}$ for a time of 10 units. Periodic boundary conditions are applied everywhere. Since the problem is well-known in the literature, we do not describe it in detail here. We report on the accuracy of the x-magnetic field of the vortex after it has completed one orbit in the computational domain, see Table I for the two-dimensional case and Table II for the three-dimensional domain. All the schemes meet their design accuracy.

TABLE I shows the accuracy analysis for the MHD vortex problem in 2D as measured in the $x$-magnetic field.

\begin{tabular}{|c|c|c|c|c|c|}
\hline Method & $\mathrm{h}$ & $L_{1}$ Error & $L_{1}$ Order & $L_{\infty}$ Error & $L_{\infty}$ Order \\
\hline \multicolumn{6}{|l|}{$\begin{array}{l}\text { ADER-WENO } \\
2^{\text {nd }} \text { Order }\end{array}$} \\
\hline & $\mathrm{h}=\mathrm{L} / 32$ & 9.6533E-01 & & $1.8442 \mathrm{E}-01$ & \\
\hline & $\mathrm{h}=\mathrm{L} / 64$ & $2.2159 \mathrm{E}-01$ & 2.12 & $4.7733 \mathrm{E}-02$ & 1.95 \\
\hline & $\mathrm{h}=\mathrm{L} / 128$ & $5.9102 \mathrm{E}-02$ & 1.91 & $1.3195 \mathrm{E}-02$ & 1.85 \\
\hline & $\mathrm{h}=\mathrm{L} / 256$ & $1.5625 \mathrm{E}-02$ & 1.92 & $4.3679 \mathrm{E}-03$ & 1.59 \\
\hline \multicolumn{6}{|l|}{$\begin{array}{l}\text { ADER-WENO } \\
3^{\text {rd }} \text { Order }\end{array}$} \\
\hline & $\mathrm{h}=\mathrm{L} / 32$ & $6.2396 \mathrm{E}-01$ & & $1.1050 \mathrm{E}-01$ & \\
\hline & $\mathrm{h}=\mathrm{L} / 64$ & $9.6015 \mathrm{E}-02$ & 2.70 & $1.9574 \mathrm{E}-02$ & 2.50 \\
\hline & $\mathrm{h}=\mathrm{L} / 128$ & $1.2064 \mathrm{E}-02$ & 2.99 & $2.5521 \mathrm{E}-03$ & 2.94 \\
\hline & $\mathrm{h}=\mathrm{L} / 256$ & $1.5101 \mathrm{E}-03$ & 3.00 & $3.3083 \mathrm{E}-04$ & 2.95 \\
\hline \multicolumn{6}{|l|}{$\begin{array}{l}\text { ADER-WENO } \\
4^{\text {th }} \text { Order }\end{array}$} \\
\hline & $\mathrm{h}=\mathrm{L} / 32$ & $8.8702 \mathrm{E}-02$ & & $1.8884 \mathrm{E}-02$ & \\
\hline & $\mathrm{h}=\mathrm{L} / 64$ & $4.7539 \mathrm{E}-03$ & 4.22 & $1.2242 \mathrm{E}-03$ & 3.95 \\
\hline & $\mathrm{h}=\mathrm{L} / 128$ & $3.0801 \mathrm{E}-04$ & 3.95 & $7.5591 \mathrm{E}-05$ & 4.02 \\
\hline & $\mathrm{h}=\mathrm{L} / 256$ & $2.3889 \mathrm{E}-05$ & 3.69 & $1.7298 \mathrm{E}-05$ & 2.13 \\
\hline
\end{tabular}

TABLE II shows the accuracy analysis for the MHD vortex problem in 3D as measured in the $x$-magnetic field.

\begin{tabular}{|c|c|c|c|c|c|}
\hline Method & $\mathrm{h}$ & $L_{1}$ Error & $L_{1}$ Order & $L_{\infty}$ Error & $L_{\infty}$ Order \\
\hline \multicolumn{6}{|l|}{$\begin{array}{l}\text { ADER-WENO } \\
2^{\text {nd }} \text { Order } \\
\end{array}$} \\
\hline & $\mathrm{h}=\mathrm{L} / 20$ & $2.0056 \mathrm{E}+00$ & & 3.1544E-01 & \\
\hline & $\mathrm{h}=\mathrm{L} / 40$ & $5.7328 \mathrm{E}-01$ & 1.81 & $1.3965 \mathrm{E}-01$ & 1.18 \\
\hline & $\mathrm{h}=\mathrm{L} / 60$ & 2.4157E-01 & 2.13 & 7.5446E-02 & 1.52 \\
\hline \multicolumn{6}{|l|}{$\begin{array}{l}\text { ADER-WENO } \\
3^{\text {rd }} \text { Order }\end{array}$} \\
\hline & $\mathrm{h}=\mathrm{L} / 20$ & $8.3151 \mathrm{E}-01$ & & $1.5921 \mathrm{E}-01$ & \\
\hline & $\mathrm{h}=\mathrm{L} / 40$ & $1.5198 \mathrm{E}-01$ & 2.45 & 3.4847E-02 & 2.19 \\
\hline
\end{tabular}




\begin{tabular}{|l|l|l|r|r|r|}
\hline & $\mathrm{h}=\mathrm{L} / 60$ & $4.6681 \mathrm{E}-02$ & $\mathbf{2 . 9 1}$ & $1.0967 \mathrm{E}-02$ & $\mathbf{2 . 8 5}$ \\
\hline $\begin{array}{l}\text { ADER-WENO } \\
4^{\text {th }} \text { Order }\end{array}$ & & & & & \\
\hline & $\mathrm{h}=\mathrm{L} / 20$ & $3.1118 \mathrm{E}-01$ & & $5.6388 \mathrm{E}-02$ & \\
\hline & $\mathrm{h}=\mathrm{L} / 40$ & $1.5365 \mathrm{E}-02$ & $\mathbf{4 . 3 4}$ & $4.5475 \mathrm{E}-03$ & $\mathbf{3 . 6 3}$ \\
\hline & $\mathrm{h}=\mathrm{L} / 60$ & $2.9836 \mathrm{E}-03$ & $\mathbf{4 . 0 4}$ & $1.0210 \mathrm{E}-03$ & $\mathbf{3 . 6 8}$ \\
\hline $\begin{array}{l}\text { ADER-WENO } \\
5^{\text {th }} \text { Order }\end{array}$ & & & & & \\
\hline & $\mathrm{h}=\mathrm{L} / 20$ & $2.9646 \mathrm{E}-01$ & & $5.8322 \mathrm{E}-02$ & \\
\hline & $\mathrm{h}=\mathrm{L} / 40$ & $6.7662 \mathrm{E}-03$ & $\mathbf{5 . 4 5}$ & $1.8454 \mathrm{E}-03$ & $\mathbf{4 . 9 8}$ \\
\hline & $\mathrm{h}=\mathrm{L} / 60$ & $8.7482 \mathrm{E}-04$ & $\mathbf{5 . 0 5}$ & $2.4588 \mathrm{E}-04$ & $\mathbf{4 . 9 7}$ \\
\hline
\end{tabular}

\section{VIII) Test Problems}

In all the following test problems, as well as in the previous accuracy analysis, we have always used the self-adjusting and positivity preserving flattener strategy outlined by Balsara in [20].

\section{VIII.a) Rotor Problem in Two-Dimensions}

This well-known MHD problem was first documented in Balsara \& Spicer [6] and also Balsara [8]. It consists of a central, uniformly rotating vortex in a non-rotating ambient medium. An initially uniform magnetic field threads through both regions. The details are described in the above-mentioned references. A circular region with radius 0.5 was triangulated using 71046 elements of characteristic size $h=0.005$. A third order divergence-free ADER-WENO scheme was used with a CFL of 0.95. The MuSIC Riemann solver was used with a one-dimensional HLLD Riemann solver in the side panels. Fig. 4 shows the final results for the rotor problem, at a time of 0.25 . Figs. $4 a, 4 b, 4 c$ and $4 d$ show the density, pressure, Mach number and magnetic pressure at the final time. All the requisite MHD flow features are nicely captured in our simulations.

\section{VIII.b) Orzag Tang Problem in Two-Dimensions}

This well-known problem by Orszag \& Tang [58] was initialized on a periodic domain spanning $[0,2 \pi] \times[0,2 \pi]$. It was run to a stopping time of $t=3.0$ with a third order accurate divergence-free ADER-WENO scheme using a CFL of 0.95 and an unstructured triangular mesh of 90126 elements of characteristic mesh size $\mathrm{h}=0.03$. The MuSIC Riemann solver was used with a one-dimensional HLLD Riemann solver in the side panels. Figs. 5a, 5b, 5c and 5d show the density, pressure, Mach number and magnetic pressure at the final time. The simulation forms a current sheet with oppositely oriented x-components of magnetic field in the center of the computational domain, as can be surmised from Fig. 5d. The velocity field also shows fluid squirting out in the positive and negative $\mathrm{x}$-directions at the location of the current sheet. 


\section{VIII.c) Propagation of Finite Amplitude Torsional Alfven Waves in Two-Dimensions}

In several fields, like astrophysics or space physics, one is interested in the evolution of waves to simulate certain problems such as turbulence. The ability to propagate finite amplitude Alfven waves over large distances and long times on a computational mesh is crucial for carrying out simulations of MHD turbulence. If the Alfven waves are damped strongly because of inherent numerical dissipation in a code, the code will fail to capture the resulting turbulence. This is because MHD turbulence is mainly sustained by Alfven waves. The Alfven wave decay test problem, first presented by Balsara [8], examines the numerical dissipation of torsional Alfven waves in two dimensions. In this test problem torsional Alfven waves propagate at an angle of $9.462^{\circ}$ to the $y$-axis through a domain given by $[-3,3]$ x $[-3,3]$. The domain was set up with a two-dimensional triangular mesh of characteristic size $h=0.05$. Periodic boundary conditions have been applied everywhere. The ratio of specific heats was chosen as $\gamma=1.4$, the reference density was set to $\rho_{0}=1$ and a background pressure of $p_{0}=2$ has been used. We do not present the remaining details of the set-up, because the problem is already well-described in the above-mentioned reference. The simulation was stopped at 129 time units by which time the Alfven waves had crossed the domain several times.

We performed one set of simulations where this problem was run with second, third and fourth order divergence-free ADER-WENO schemes and the MuSIC Riemann solver. Since this problem is subsonic, the strongly interacting state plays a major role in the evolution of Alfven waves. The problem was run with a multidimensional Riemann solver that utilized a onedimensional HLLD Riemann solver that was patterned after the work of Miyoshi \&Kusano [74].

Fig. 6a shows the evolution of the maximum z-velocity in the torsional Alfven wave as a function of time. Fig. $6 \mathrm{~b}$ shows the evolution of the maximum z-magnetic field in the torsional Alfven wave as a function of time. For Fig. 6 we used second, third and fourth order divergencefree ADER-WENO schemes. We see that the fourth order ADER-WENO scheme has the least numerical dissipation while the second order ADER-WENO scheme had the most dissipation, as expected.

\section{VIII.e) MHD Blast Wave Problem in Two and Three Space-Dimensions}

Here we solve a two-dimensional and a three-dimensional variant of a problem that was originally presented in Balsara \& Spicer [6]. The plasma $\beta$ measures the ratio of the thermal pressure to the magnetic pressure. As the plasma's $\beta$ becomes smaller, this problem becomes increasingly stringent. The problem consists of a $\gamma=1.4$ gas with unit density and a pressure of 0.1 initialized on a circular two-dimensional or a spherical three-dimensional computational domain with radius $\mathrm{R}=0.5$. For the $2 \mathrm{D}$ setup we use an initially constant magnetic field of $\mathbf{B}=(70,0,0)$, while in the $3 \mathrm{D}$ case we use $\mathbf{B}=50 / \sqrt{3}(1,1,1)$. The pressure is reset to a value of 1000 inside a central region with a radius of 0.1 . The plasma's $\beta$ is initially given by $1.005 \times 10^{-3}$ 
for the three-dimensional problem. A CFL number of 0.45 was used for the $2 \mathrm{D}$ simulations and 0.3 for the $3 \mathrm{D}$ computations. The problem is run up to a time of 0.012 , by which time a strong magnetosonic blast wave propagates through the domain. The problem was run with a third order divergence-free ADER-WENO scheme and a MuSIC Riemann solver with linear variation in the similarity variables. The $2 \mathrm{D}$ simulations have been carried out on a triangular mesh composed of 71046 elements with characteristic size $h=1 / 200$. For the $3 \mathrm{D}$ computations we have used an unstructured tetrahedral mesh of 422468 elements with a characteristic mesh size of $h=0.02$.

First we present the two-dimensional results. Figs. 7a, 7b, 7c and 7d show the plots for density, pressure, velocity magnitude and magnetic field magnitude, respectively. We see that the densities and pressures are positive, as expected. The previously-cited positivity preserving methods are very useful in that regard. Figs. $8 \mathrm{a}, 8 \mathrm{~b}, 8 \mathrm{c}$ and $8 \mathrm{~d}$ contain the results obtained for the same quantities in the three dimensional case within the $z=0$ slice plane. A sketch of the unstructured tetrahedral mesh together with the iso-contour surfaces of the magnetic field intensity are depicted in Fig. 9.

\section{VIII.d) MHD Field Loop Advection Problem in Two and Three Space-Dimensions}

This problem is first set up on a two-dimensional domain that spans $[-1,1] \times[-0.5,0.5]$ and that has been discretized using 45008 triangular elements of characteristic mesh size $\mathrm{h}=1 / 100$. The problem consists of a two-dimensional loop of magnetic field with a very low magnetic pressure compared to the gas pressure. The magnetic pressure is constant inside the loop and falls abruptly to zero at the loop's boundary which is initially set up at a radius of 0.3 units. The details of the set-up are described in Gardiner \& Stone [43] and are not repeated here. The problem was run to a final time of $\mathrm{t}=1.0$ with a third and fourth order divergence-free ADERWENO scheme and the MuSIC Riemann solver. The multidimensional Riemann solver used a one-dimensional HLLD Riemann solver and had linear variation in the similarity variables. A CFL number of 0.95 was used and no doubling of the numerical dissipation was seen to be necessary when evaluating the edge-centered electric fields. The resulting magnitudes of the magnetic field are shown in Fig. 10a and 10b for the third and fourth order cases. From Fig. 10 we see that the loop's profile is almost perfectly isotropic, owing to the use of the multidimensional Riemann solver technology in combination with high order divergence-free finite volume schemes on unstructured meshes. The fourth order results are sharper than the third order results, as expected.

We now run the same test case again on a three-dimensional periodic domain [$0.5,0.5] \times[-0.5,0.5] \times[-0.5,0.5]$, discretized by $1,772,044$ tetrahedral elements with characteristic mesh size $h=1 / 64$. The velocity vector of this advection problem is given by $\mathbf{v}=(1,1,0)$ and the final result is shown at a time of unity so that the field loop returns to its original location. In the case of unstructured tetrahedral meshes the field loop is in general not aligned with the element faces, hence it is not necessary to tilt the 3D field loop as in [44]. A third order divergence-free ADER-WENO scheme and the MuSIC Riemann solver were used for this simulation. The 
unstructured mesh used for our computations is displayed in Fig. 11a. A three-dimensional isocontour surface plot of the magnetic field intensity is drawn in Fig. 11b. A two-dimensional cut through our computational results is presented in Fig. 11c. We find that the isotropic shape of the field loop is perfectly preserved after one periodic passage through the computational domain.

\section{IX) Conclusions}

Numerical MHD serves as a prototype for a large class of very useful mimetic partial differential equations. This justifies the interest in developing high quality numerical algorithms for this system of equations. Staggered mesh formulations for MHD that draw on the dualism between electric fields and Godunov fluxes have already been developed for structured meshes (Balsara \& Spicer [6]). In recent years it has been realized that there are two essential new innovations that are needed for divergence-free MHD simulations on structured meshes. The first new innovation is the divergence-free reconstruction of magnetic fields at all orders (Balsara [7], [8], [9]). The second new innovation is the development of multidimensional Riemann solvers (Balsara [15], [16], [18]) along with the realization in these papers that the multidimensional Riemann problem provides the correct multidimensional upwinding that is needed for the edgecentered electric fields. Analogous multidimensional Riemann solvers that are suitable for unstructured meshes have also been developed (Balsara, Dumbser \& Abgrall [17], Balsara \& Dumbser [19]). Positivity preserving schemes for MHD have also been developed (Balsara \& Spicer [5], Balsara [20]) which lend an additional measure of robustness to structured or unstructured mesh MHD calculations. To achieve full geometric versatility, it is only natural that one should want the same advances in numerical MHD to be made for unstructured meshes.

In this paper we have presented a novel strategy for carrying out high order accurate divergence-free calculations of MHD flow on unstructured triangular and tetrahedral meshes in two and three space dimensions. Our new divergence-free reconstruction operator is based on a staggered mesh for the facially-averaged magnetic field components, together with a constrained $\mathrm{L}_{2}$ projection strategy that can be easily implemented as a post-processor of an existing, classical finite volume WENO reconstruction algorithm based on cell averages. Results for such divergence-free WENO reconstruction schemes with second, third, fourth and fifth order of accuracy have been presented. We have also shown the value of using multidimensional Riemann solvers for obtaining the electric field. In particular, the MuSIC Riemann solver has been shown to work very well for unstructured mesh MHD calculations. It is also shown that ADER timestepping works very well when it is coupled with divergence-free WENO reconstruction of the magnetic fields and a genuinely multidimensional Riemann solver. Several stringent test problems are shown to operate well on two-dimensional and three-dimensional unstructured meshes. While we have proved the applicability of our method to triangulated and tetrahedral meshes, Balsara [8], [9] has also shown its applicability to hexahedral meshes. We can therefore safely conjecture that the method might have applicability to engineering applications where the elements might be combinations of tetrahedrons, prisms and pyramids. 
In this paper we have focused on finite volume, WENO-based reconstruction methods for divergence-free MHD which are based on multidimensional Riemann solvers. The design of multidimensional Riemann solvers is now well-understood (Balsara [15], [16], [18], Balsara, Dumbser \& Abgrall [17], Balsara \& Dumbser [19]) and we see that MuSIC Riemann solvers play a central role in the update of the magnetic field. Higher order timestepping with a simple one-step structure has also become a reality with the advent of the family of high order ADER schemes (Titarev \& Toro [70], [71], Toro \& Titarev [72], Dumbser et al. [36], [38], Balsara et al. [13], [14]). The whole field of higher order DG and PNPM schemes has opened up, with nonlinearly-stabilized schemes that have now become a reality (Diot, Clain \& Loubere [34], Loubere, Dumbser \& Diot [55], Dumbser et al. [40]). Consequently, this paper is but a first step in the development of advanced higher order constraint-preserving methods for unstructured meshes that use these very important computational innovations.

\section{Acknowledgements}

DSB acknowledges support via NSF grants NSF-AST-1009091, NSF-ACI-1307369 and NSF-DMS-1361197. DSB also acknowledges support via NASA grants from the Fermi program as well as NASA-NNX 12A088G. Several simulations were performed on a cluster at UND that is run by the Center for Research Computing. Computer support on NSF's XSEDE and Blue Waters computing resources is also acknowledged.

MD was funded by the European Research Council (ERC) under the European Union's Seventh Framework Programme (FP7/2007-2013) with the research project STiMulUs, ERC Grant agreement no. 278267. The authors acknowledge PRACE for awarding access to the SuperMUC supercomputer of the Leibniz Rechenzentrum (LRZ) in Munich, Germany.

Last but not least we would also like to thank the two anonymous referees for their very constructive comments and suggestions, which helped to improve the clarity and the readability of this manuscript. 


\section{References}

[1] R. Abgrall, On essentially non-oscillatory schemes on unstructured meshes : Analysis and implementation, J. Comput. Phys., 114 (1994) 45-58

[2] J. Balbas, E. Tadmor and C.-C.Wu, Non-oscillatory central scheme for one- and twodimensional MHD equations, J. Comput. Phys., 201 (2004) 261-285

[3] D. S. Balsara, Linearized formulation of the Riemann problem for adiabatic and isothermal magnetohydrodynamics, Astrophysical Journal Supplement 116 (1998) 119

[4] D.S. Balsara, Total variation diminishing algorithm for adiabatic and isothermal magnetohydrodynamics, Astrophysical Journal Supplement 116 (1998) 133

[5] D. S. Balsara and D. S. Spicer, Maintaining pressure positivity in magnetohydrodynamic simulations, Journal of Computational Physics 148 (1999) 133-148

[6] D. S. Balsara and D. S. Spicer, A staggered mesh algorithm using high order Godunov fluxes to ensure solenoidal magnetic fields in magnetohydrodynamic simulations, Journal of Computational Physics 149 (1999) 270-292

[7] D.S. Balsara ,Divergence-free adaptive mesh refinement for magnetohydrodynamics, Journal of Computational Physics 174 (2001) 614-648

[8] D. S. Balsara, Second-order-accurate schemes for magnetohydrodynamics with divergencefree reconstruction, Astrophysical Journal Supplement 151 (2004) 149-184

[9] D.S. Balsara, Divergence-free reconstruction of magnetic fields and WENO schemes for magnetohydrodynamics, J. Comput. Phys., 228 (2009) 5040-5056

[10] D.S. Balsara\& J.S. Kim, An Intercomparison Between Divergence-Cleaning and Staggered Mesh Formulations for Numerical Magnetohydrodynamics, Astrophysical Journal 602 (2004) 1079

[11] D. S. Balsara and C.-W. Shu, Monotonicity Preserving Weighted Non-oscillatory schemes wirh increasingly High Order of Accuracy, Journal of Computational Physics 160 (2000) 405452

[12] D. S. Balsara, C. Altmann, C.D. Munz, M. Dumbser, A sub-cell based indicator for troubled zones in RKDG schemes and a novel class oh hybrid RKDG+HWENO schemes, Journal of Computational Physics 226 (2007) 586- 620 
[13] D.S. Balsara, T. Rumpf, M. Dumbser and C.-D.Munz, Efficient, high accuracyADERWENO schemes for hydrodynamics and divergence-free magnetohydrodynamics, Journal of Computational Physics 228 (2009) 2480-2516

[14] Balsara, D.S., Dumbser, M., Meyer, C., Du, H. \& Xu, Z., Efficient Implementation of ADER schemes for Euler and Magnetohydrodynamic flow on structured meshes - Comparison with Runge-Kutta methods, Journal of Computational Physics, 235 (2013) 934-969

[15] D.S. Balsara, Multidimensional HLLE Riemann solver; Application to Euler and Magnetohydrodynamic Flows, J. Comput. Phys., 229 (2010) 1970-1993

[16] D.S. Balsara, A two-dimensional HLLC Riemann solver for conservation laws: Application to Euler and magnetohydrodynamic flows, Journal of Computational Physics 231 (2012) 74767503

[17] D.S. Balsara, M. Dumbser and R. Abgrall, Multidimensional HLL and HLLC Riemann Solvers for Unstructured Meshes - With Application to Euler and MHD Flows, Journal of Computational Physics 261 (2014) 172-208

[18] D.S. Balsara, Multidimensional Riemann Problem with Self-Similar Internal Structure Part I - Application to Hyperbolic Conservation Laws on Structured Meshes, to appear, Journal of Computational Physics (2014)

[19] D.S. Balsara\& M. Dumbser, Multidimensional Riemann Problem with Self-Similar Internal Structure - Part II - Application to Hyperbolic Conservation Laws on Unstructured Meshes, submitted, Journal of Computational Physics (2014)

[20] Balsara, D.S., Self-Adjusting, Positivity Preserving High Order Schemes for Hydrodynamics and Magnetohydrodynamics, Journal of Computational Physics, 231 (2012) 7504-7517

[21] W. Boscheri, M. Dumbser and M. Righetti, A semi-implicit scheme for $3 D$ free surface flows with high-order velocity reconstruction on unstructured Voronoi meshes, International Journal for Numerical Methods in Fluids, 72 (2013) 607-631

[22] J. U. Brackbill and D. C. Barnes, The effect of nonzero $\nabla \cdot \boldsymbol{B}$ on the numerical solution of the magnetohydrodynamic equations, Journal of Computational Physics 35 (1980) 426-430

[23] S. H. Brecht, J. G. Lyon, J. A. Fedder, K. Hain, A simulation study of east-westIMF effects on the magnetosphere, Geophysical ReserachLett. 8 (1981) 397

[24] M. Brio \& C.C. Wu ,An upwind differencing scheme for the equations of ideal magnetohydrodynamics, Journal of Computational Physics 75 (1988) 400

[25] P. Cargo \& G. Gallice, Roe matrices for ideal MHD and systematic construction of Roe matrices for systems of conservation laws, J. Comput. Phys., 136 (1997) 446 
[26] Y. Cheng, F. Li, J. Qiu \& L. Xu, Positivity-preserving DG and central DG methods for ideal MHD equations, J. Comput. Phys., 238 (2013) 255

[27] A.J. Christlieb, J.A. Rossmanith and Q. Tang, Finite difference weighted essentially nonoscillatory schemes with constrained transport for ideal magnetohydrodynamics, J. Comput. Phys., 268 (2014) 302-325

[28] B. Cockburn F. Li and C.-W. Shu, Locally divergence-free discontinuousGalerkin method for the Maxwell equations, Journal of Computational Physics 141 (2005) 413-442

[29] R. K. Crockett, P. Colella, R. T. Fisher, R. I. Klein \& C. F. McKee, An unsplitcell-centered Godunov method for ideal MHD, Journal of Computational Physics 203 (2005) 422

[30] W. Dai and P.R. Woodward, An approximate Riemann solver for ideal magnetohydrodynamics, Journal of Computational Physics 111 (1994) 354-372

[31] W. Dai and P.R. Woodward, On the divergence-free condition and conservationlaws in numerical simulations for supersonic magnetohydrodynamic flows, Astrophysical Journal 494 (1998) 317-335

[32] A. Dedner, F. Kemm, D. Kröner, C.-D.Munz, T. Schnitzer, M. Wesenberg, Hyperbolic divergence cleaning for MHD equations, Journal of Computational Physics 175 (2002) 645-673

[33] C. R. DeVore, Flux-corrected transport techniques for multidimensional compressible magnetohydrodynamics, Journal of Computational Physics 92 (1991) 142-160

[34] S. Diot, S. Clain \& R. Loubere, Improved detection criteria for the Multi-dimensional Optimal Order Detection (MOOD) on unstructured meshes with very high-order polynomials, Computers \& Fluids 64 (2012) 43-63

[35] M. Dubiner, Spectral methods on triangles and other domains, Journal of Scientific Computing, 6 (1991) 345-390.

[36] M. Dumbser, D. Balsara, E.F. Toro, C.D. Munz, A unified framework for the construction of one-step finite volume and discontinuous Galerkin schemes on unstructured meshes, Journal of Computational Physics 227 (2008) 8209-8253

[37] M. Dumbser, M. Käser, V.A. Titarev\& E.F. Toro, Quadrature-free non-oscillatory finite volume schemes on unstructured meshes for nonlinear hyperbolic systems, Journal of Computational Physics 226 (2007) 204-243

[38] M. Dumbser, O. Zanotti, A. Hidalgo and D.S. Balsara, ADER-WENO Finite Volume Schemes with Space-Time Adaptive Mesh Refinement, J. Comp. Phys. Vol. 248, Pgs. 257-286 (2013) 
[39] M. Dumbser \& M. Käser, Arbitary high order non-oscillatory finite volume schemes on unstuructured meshes for linear hyperbolic systems, Journal of Computational Physcis, 221 (2007) 693-723

[40] M. Dumbser, O. Zanotti, R. Loubere, S. Diot, A Posteriori Subcell Limitation of the Discontinuous Galerkin Finite Element Method for Hyperbolic Conservation Laws, to appear, Journal of Computational Physcis, (2014)

[41] C.R.Evans and J.F.Hawley, Simulation of Magnetohydrodynamic Flows: A Constrained Transport Method, Astrophysical Journal 332 (1989) 659

[42] S. A. E. G. Falle, S. S. Komissarov and P. Joarder, A multidimensional upwind scheme for magnetohydrodynamics, Monthly Notices of the Royal Astronomical Society 297 (1998), 265-277

[43] T. Gardiner \& J.M. Stone, An unsplit Godunov method for ideal MHD via constrained transport, Journal of Computational Physics, 205 (2005), 509

[44] T. Gardiner \& J.M. Stone, An unsplit Godunov method for ideal MHD via constrained transport in three dimensions, Journal of Computational Physics, 227 (2008), 4123

[45] K.F. Gurski, An HLLC-type approximate Riemann solver for ideal magnetohydrodynamics, SIAM J. Sci. Comput. 25 (2004) 2165

[46] C. Hu and C.-W. Shu,Weighted essentially non-oscillatory schemes on triangular meshes, J. Comput. Phys., 150 (1999) 97-127

[47] A. Jeffrey and T. Taniuti, Nonlinear Wave Propagation, New York Academic, (1964)

[48] G.-S. Jiang and C.-W. Shu, Efficient implementation of weighted ENO schemes,Journal of Computational Physics 126 (1996) 202-228

[49] G.-S. Jiang \& C.C. Wu, A high-order WENO finite difference scheme for the equations of ideal magnetohydrodynamics, J. Comput. Phys., 150(2) (1999) 561-594

[50] D. Lee, A solution accurate, efficient and stable unsplit staggered mesh scheme for three dimensional magnetohydrodynamics, J. Comput. Phys., 243 (2013) 269-292

[51] F. Li, L. Xu and S. Yakovlev,Central discontinuous Galerkin methods for ideal MHD equations with the exactly divergence-free magnetic field, J. Comput. Phys., 230(12) (2011) 4828-4847

[52] F. Li and C.-W. Shu, Locally divergence-free discontinuous Galerkin methods for MHD equations, Journal of Scientific Computing, 22-23 (2005) 413-442 
[53] S. Li, High order central scheme on overlapping cells for magneto- hydrodynamic flows with and without constrained transport method, J. Comput. Phys., 227(15) (2008) 7368-7393

[54] P. Londrillo and L. DelZanna, On the divergence-free condition in Godunov-type schemes for ideal magnetohydrodynamics: the upwind constrained transport method, Journal of Computational Physics 195 (2004) 17-48

[55] R. Loubere, M. Dumbser \& S. Diot, A New Family of High Order Unstructured MOOD and ADER Finite Volume Schemes for Multidimensional Systems of Hyperbolic Conservation Laws, Communication in Computational Physics, 2014. in press

[56] T. Miyoshi and K. Kusano, A multi-state HLL approximate Riemann solver for ideal magnetohydrodynamics, J. Comput. Phys., 208 (2005) 315-344

[57] P. Mocz, M. Vogelsberger\& L. Hernquist, A constrained transport scheme for MHD on Unstructured and moving meshes, to appear, Monthly Notices of the Royal Astronomical Society (2014)

[58] S. A. Orszag and C. M. Tang, Small-scale structure of two-dimensional magnetohydrodynamic turbulence, Journal of Fluid Mechanics, 90 (1979) 129

[59] Powell, K.G. An Approximate Riemann Solver for MHD ( that actually works in more than one dimension), ICASE Report No. 94-24, Langley VA, (1994)

[60] J. Proriol, Sur une famille de polynomes 'a deux variables orthogonaux dans un triangle, C. R. Acad.Sci. Paris 257 (1957) 2459-2461

[61] P. L. Roe and D. S. Balsara, Notes on the eigensystem of magnetohydrodynamics, SIAM Journal of applied Mathematics 56 (1996), 57

[62] D. Ryu and T.W. Jones, Numerical MHD in astrophysics: algorithm and tests for onedimensional flow, Astrophysical Journal 442 (1995) 228

[63] D. Ryu, F. Miniati, T. W. Jones, and A. Frank, A divergence-free upwind code for multidimensional magnetohydrodynamic flows, Astrophysical Journal 509 (1998) 244-255

[64]C.W. Schulz-Rinne, J.P. Collins, and H.M. Glaz, Numerical solution of the Riemann problem for two-dimensional gas dynamics, SIAM J. Sci. Comput., 14(7) (1993) 1394-1414

[65] S.J. Sherwin and G.E. Karniadakis A triangular spectral element method.Applications to the incompressible Navier-Stokes equations, Comput.Meth. Appl. Mech. Engrg. 123 (1995) 189229.

[66] C.-W. Shu and S. J. Osher, Efficient implementation of essentially non-oscillatory shock capturing schemes, Journal of Computational Physics 77 (1988) 439-471 
[67] C.-W. Shu and S. J. Osher, Efficient implementation of essentially non-oscillatory shock capturing schemes II, Journal of Computational Physics 83 (1989) 32-78

[68] A.Taube, M. Dumbser, D.S, Balsara \& C.D. Munz, Arbitrary High Order Discontinuous Galerkin Schemes for the MHD Equations, SIAM J. Scientific Computing, Vol. 30(3) (2007) 441-461

[69] G. Toth, The $\nabla \square \mathbf{B}=0$ Constraint in Shock-Capturing MHD Codes, J. Comput. $\quad$ Phys., vol. 161, pg. 605, (2000)

[70] V.A. Titarev, and E.F.Toro, ADER: arbitrary high order Godunov approach, Journal of Scientific Computing 17 (1-4) (2002) 609-618

[71] V.A. Titarev and E.F.Toro, ADER schemes for three-dimensional nonlinear hyperbolic systems, Journal of Computational Physics, 204 (2005) 715-736

[72] E.F. Toro, and V.A. Titarev, Solution of the generalized Riemann problem for advection reaction equations, Proceedings of the Royal Society of London, Series A 458 (2002) 271-281

[73] Z. Xu, D. S. Balsara \& H. Du, Divergence-free WENO Reconstruction-based finite volume scheme for solving ideal MHD equations on triangular meshes, submitted, Communications in Computational Physics (2014)

[74] K.S. Yee, Numerical Solution of Initial Boundary Value Problems Involving Maxwell Equation in an Isotropic Media, IEEE Trans. Antenna Propagation 14 (1966) 302

[75] A.L. Zachary, A. Malagoli \& P. Colella, A higher-order Godunov method for multidimensional ideal Magnetohydrodynamics, SIAM J. Sci. Comput., 15 (1994) 263

[76] A.H. Stroud, Approximate Calculation of Multiple Integrals, Prentice-Hall Inc., Englewood Cliffs, NJ, 1971. 


\section{Figure Captions}

Fig. la shows the flowchart of operations in a traditional divergence-free ADER-WENO algorithm for $M H D$.

Fig. $1 b$ shows the flowchart of operations in our new divergence-free ADER-WENO algorithm for $M H D$.

Fig. 2 shows the vertices of a triangular element in two-dimensions. The three outward normals to the faces are also shown along with the lengths of the faces. In each face we can set up a local coordinate system with range [-1/2,1/2].

Fig. 3 The left panel shows the vertices of a tetrahedral element in three-dimensions. The four unit outward normal vectors to the faces are also shown along with the areas of the faces. The unit vectors along each of the edges are also shown. In each face we can set up a locally orthogonal coordinate system. This is shown for the triangular face $P_{1} P_{2} P_{3}$ in the right panel where the coordinate system is set up at the centroid $C_{1}$ of that face.

Figs. $4 a, 4 b, 4 c$ and $4 d$ show the density, pressure, Mach number and magnetic pressure for the MHD rotor problem at the final time. A third order divergence-free ADER-WENO scheme was used along with the MuSIC Riemann solver.

Figs. 5a, 5b, 5c and 5d show the density, pressure, Mach number and magnetic pressure for the Orzag-Tang problem. A third order divergence-free ADER-WENO scheme was used along with the MuSIC Riemann solver.

Fig. 6a shows the evolution of the maximum z-velocity in the torsional Alfven wave as a function of time. Fig. $6 b$ shows the evolution of the maximum z-magnetic field in the torsional Alfven wave as a function of time. For Fig.6 we used second, third and fourth order divergence-free ADER-WENO schemes with the MuSIC Riemann solver.

Figs. $7 a, 7 b, 7 c$ and $7 d$ show the density, pressure, velocity magnitude and magnetic field magnitude for the two-dimensional blast problem. A third order divergence-free ADER-WENO scheme was used along with the MuSIC Riemann solver.

Figs. $8 a, 8 b, 8 c$ and $8 d$ show the density, pressure, velocity magnitude and magnetic field magnitude for the three-dimensional blast problem. A third order divergence-free ADER-WENO scheme was used along with the MuSIC Riemann solver.

Fig. 9 shows a sketch of the unstructured tetrahedral mesh together with the iso-contour surfaces of the magnetic field intensity for the three-dimensional blast problem. 
Fig. 10 shows the result of the field loop advection problem in two-dimensions. The resulting magnitudes of the magnetic field are shown in Fig. 10a and 10b for the third and fourth order cases respectively. The fourth order results are sharper than the third order results, as expected.

Fig. 11 shows the result of the field loop advection problem in three-dimensions. The unstructured mesh used for our computations is displayed in Fig. 11a. A three-dimensional isocontour surface plot of the magnetic field intensity is drawn in Fig. 11b. A two-dimensional cut through our computational results is presented in Fig. 11c. We find that the isotropic shape of the field loop is perfectly preserved after one periodic passage through the computational domain. 
Reconstruction: Reconstruct high order zone-centered fluid variables from the known zone averages (requires a zone-based WENO reconstruction).

Reconstruct high order moments of the magnetic field in the faces from the known face-averaged magnetic fields (requires a face-based WENO reconstruction).

Facial averages and higher order moments of the magnetic field in the faces are used to obtain a high order divergence-free magnetic field within each zone.

Predictor: All spatial moments of fluid variables and magnetic fields are available in each zone.

Use ADER to build all needed moments in time for fluid variables \& magnetic fields within each zone.

Corrector: Invoke 1D RS at face-centers and MuSIC RS at edge-centers. Obtain high order facially-averaged fluid fluxes.

Also obtain high order edge-averaged electric fields from MuSIC RS.

Update: Use facially-averaged fluxes to update the zone-averaged fluid variables. Use edge-averaged electric fields to update the face-averaged magnetic fields, see (2.5) and (2.11). The face-averaged magnetic fields remain div-free according to (2.4) and (2.10).

Fig. 1a shows the flowchart of operations in a traditional divergence-free ADERWENO algorithm for MHD, see [14,18,73]. 
Reconstruction: Reconstruct high order zone-centered fluid variables and an auxiliary high order zonecentered magnetic field from the known zone averages (requires only one zone-based WENO reconstruction). The resulting reconstructed magnetic field within the zone is not divergence-free, but is used to obtain the higher order moments of the magnetic field in the faces.

Use the constrained L2 projection (5.5)-(5.13) to obtain a high order accurate zone-centered reconstruction of the magnetic field that is locally and globally divergence-free according to (3.1) and (3.2).

The auxiliary variables, i.e. the zone-averaged magnetic fields, are reset after the projection step, see (5.14). This makes the zone-averaged magnetic fields consistent with the face-averaged magnetic fields.

Predictor: All spatial moments of fluid variables \& magnetic fields are available in each zone. Use ADER to build all needed moments in time for fluid variables \& magnetic fields within each zone.

Corrector: Invoke 1D RS at face-centers and MuSIC RS at edge-centers.

Obtain high order face-averaged fluid fluxes \& face-averaged magnetic fluxes, which are needed for updating the helping variables, i.e. auxiliary zone-averaged magnetic fields.

Also obtain high order edge-averaged electric fields from MuSIC RS.

Update: Use face-averaged fluid fluxes to update the zone-averaged fluid variables.

Also use facially-averaged magnetic fluxes to update the auxiliary zone-averaged magnetic fields, i.e. the helping variables.

Use edge-averaged electric fields to update the face-averaged magnetic fields, see (2.5) and (2.11). The face-averaged magnetic fields remain div-free according to (2.4) and (2.10).

Fig. $1 b$ shows the flowchart of operations in our new divergence-free ADER-WENO algorithm for MHD. 


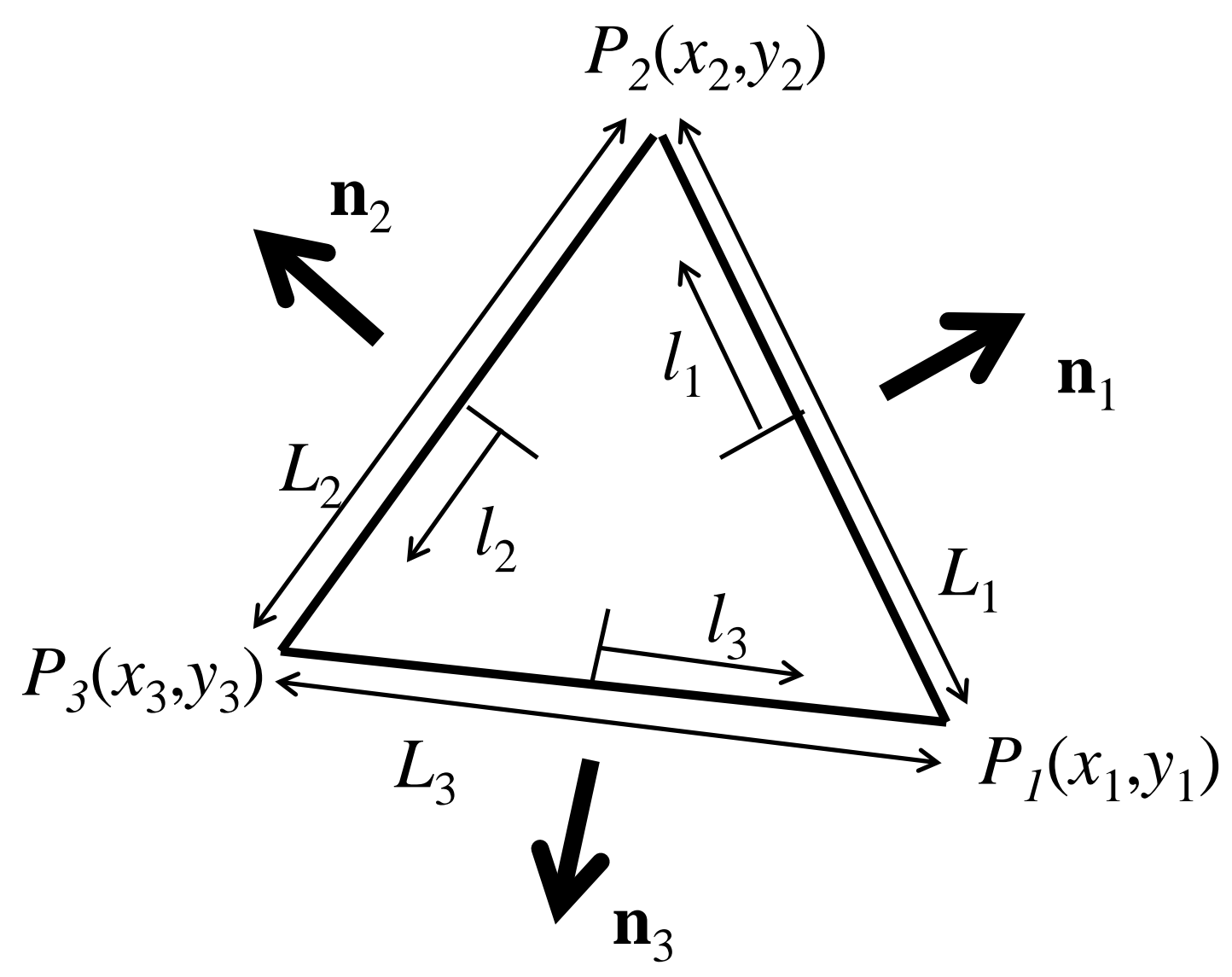

Fig. 2 shows the vertices of a triangular element in two-dimensions. The three outward normals to the faces are also shown along with the lengths of the faces. In each face we can set up a local coordinate system with range [-1/2,1/2]. 


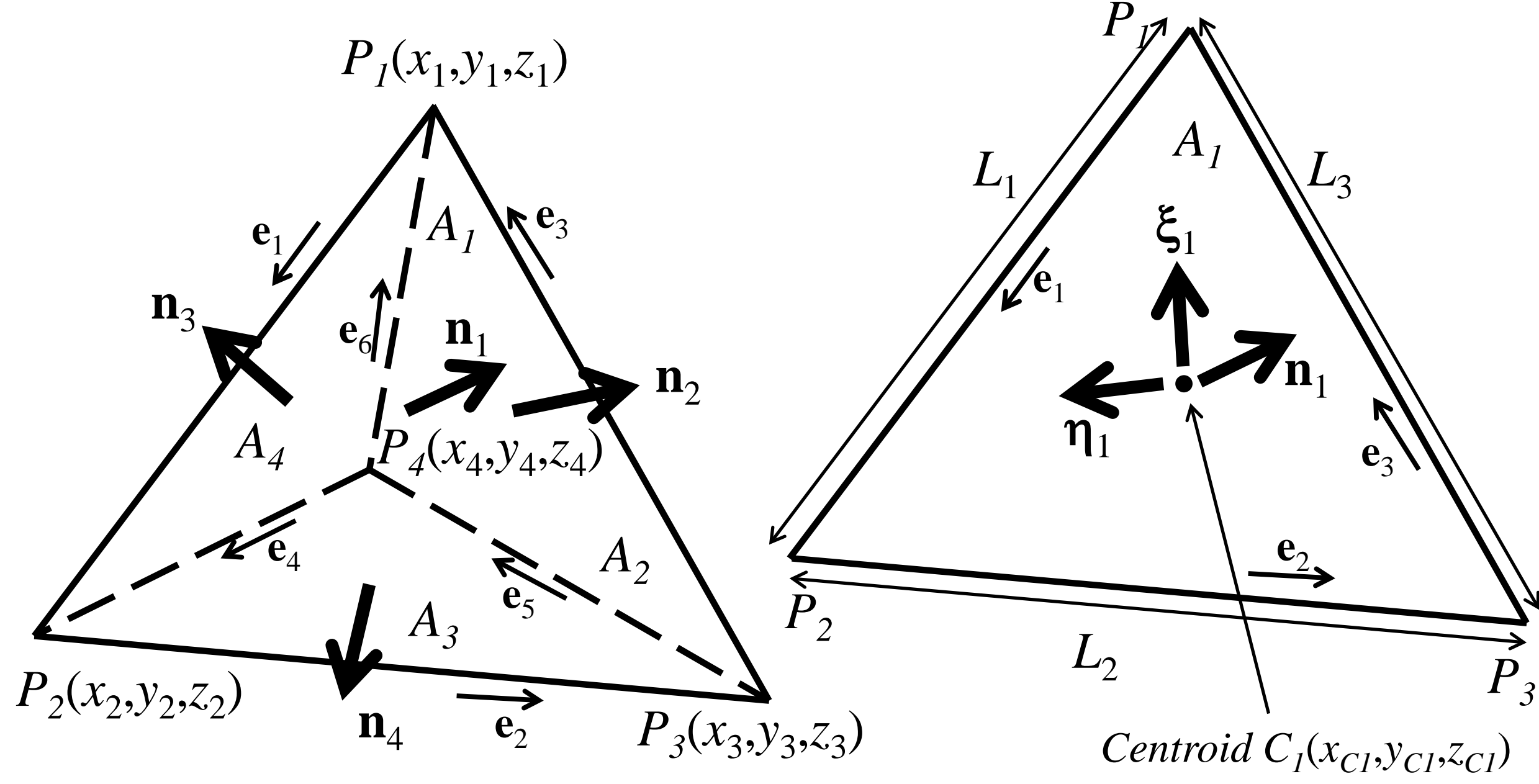

Fig. 3 The left panel shows the vertices of a tetrahedral element in three-dimensions. The four unit outward normal vectors to the faces are also shown along with the areas of the faces. The unit vectors along each of the edges are also shown. In each face we can set up a locally orthogonal coordinate system. This is shown for the triangular face $P_{1} P_{2} P_{3}$ in the right panel where the coordinate system is set up at the centroid $C_{1}$ of that face. 
a)

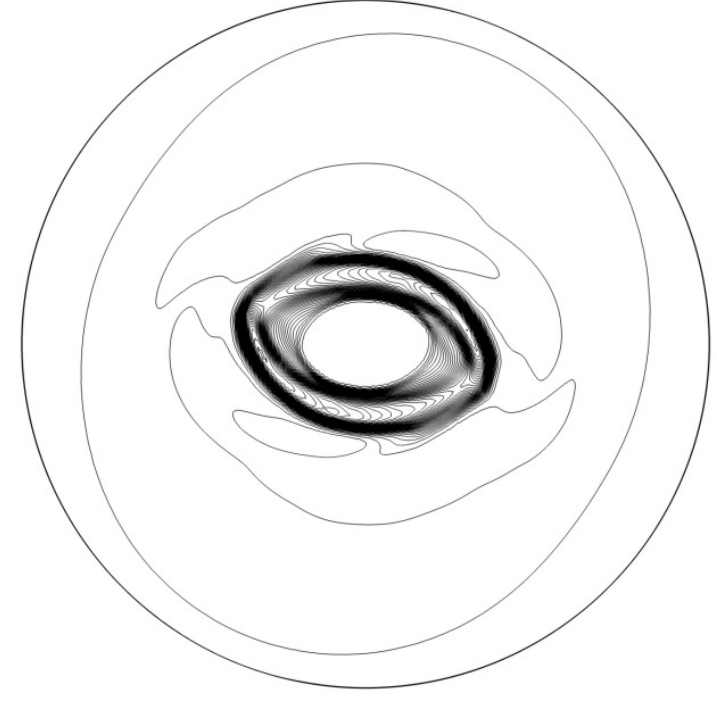

c)

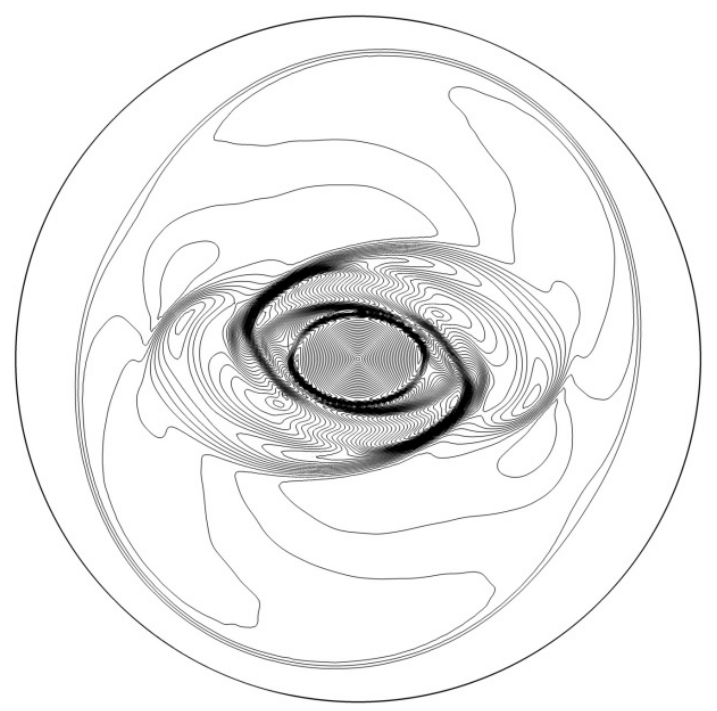

b)

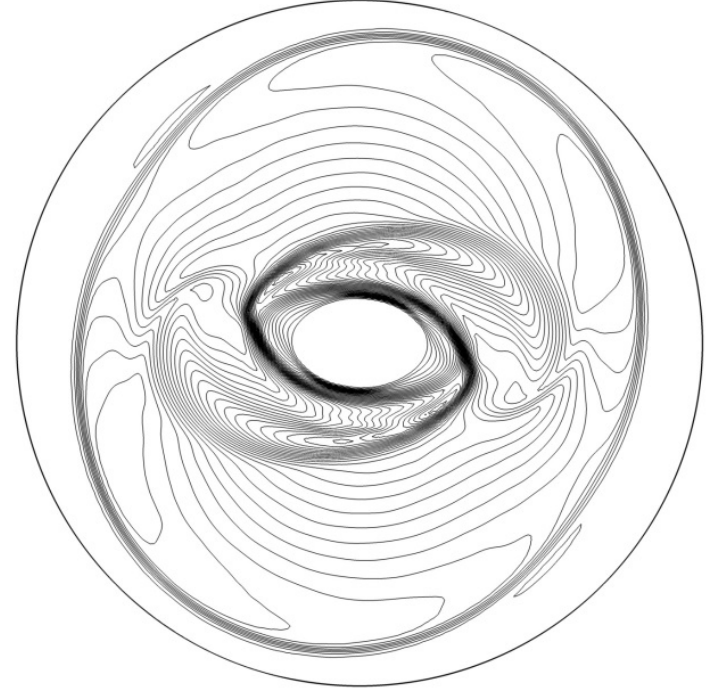

d)

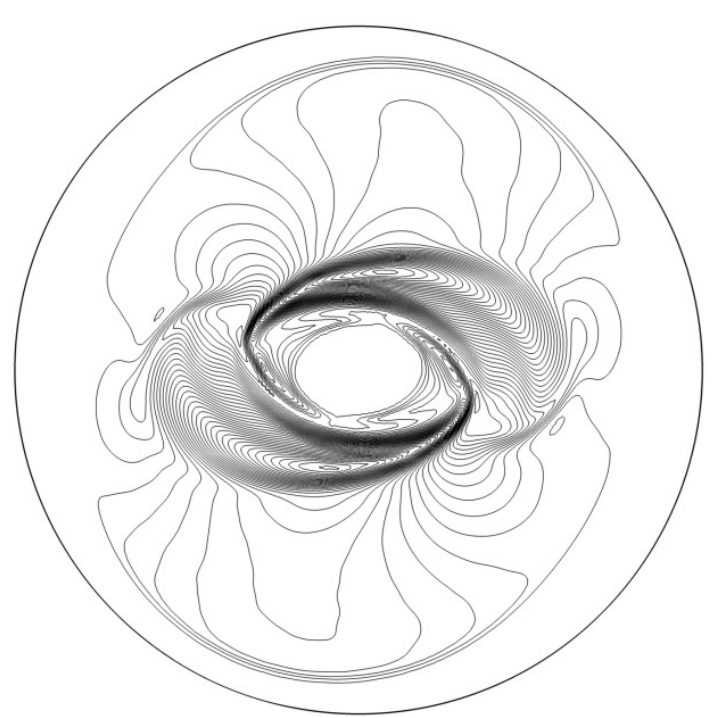

Figs. $4 a, 4 b, 4 c$ and $4 d$ show the density, pressure, Mach number and magnetic pressure for the MHD rotor problem at the final time. A third order divergence-free ADERWENO scheme was used along with the MuSIC Riemann solver. 
a)

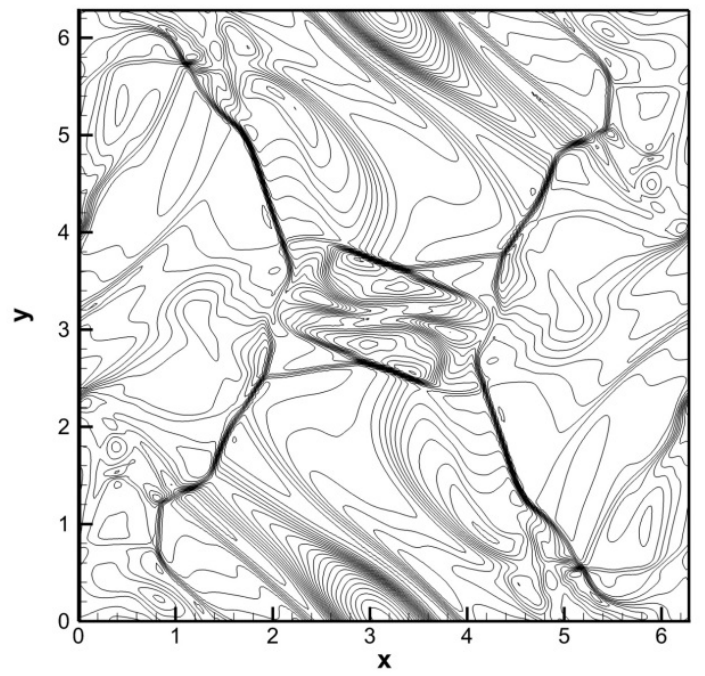

c)

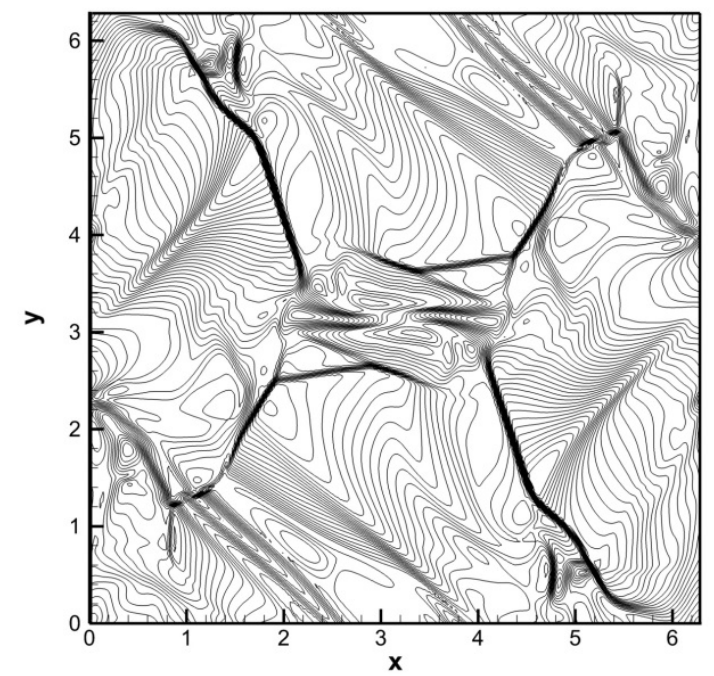

b)

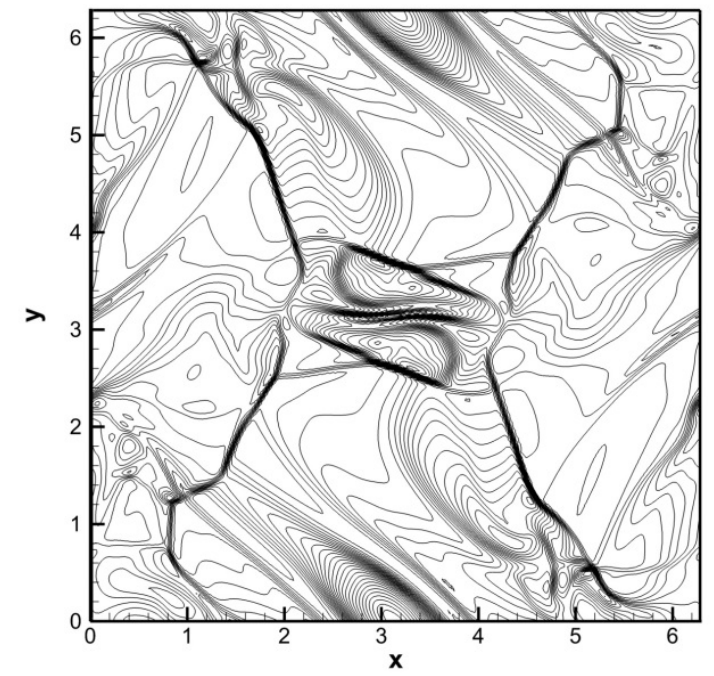

d)

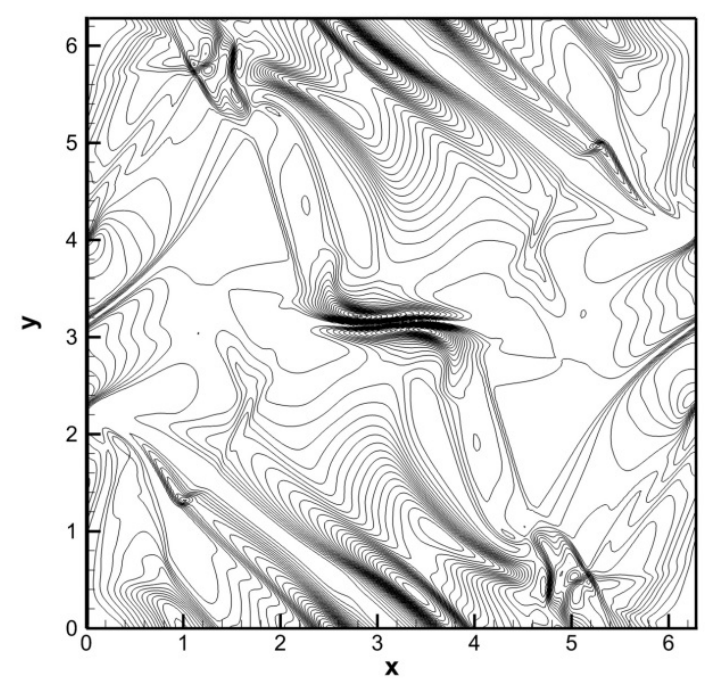

Figs. $5 a, 5 b, 5 c$ and $5 d$ show the density, pressure, Mach number and magnetic pressure for the Orzag-Tang problem. A third order divergence-free ADER-WENO scheme was used along with the MuSIC Riemann solver . 

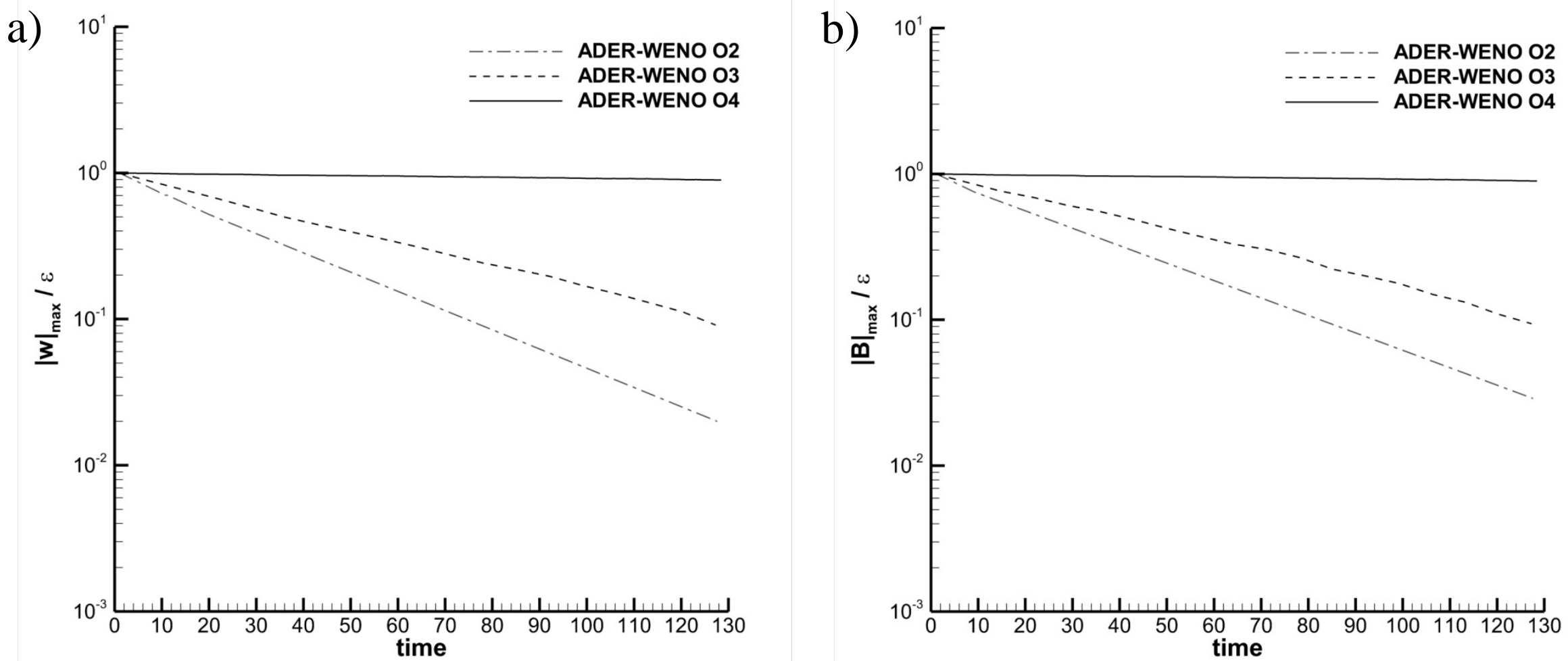

Fig. 6a shows the evolution of the maximum z-velocity in the torsional Alfven wave as a function of time. Fig. $6 b$ shows the evolution of the maximum z-magnetic field in the torsional Alfven wave as a function of time. For Fig. 6 we used second, third and fourth order divergence-free ADER-WENO schemes with the MuSIC Riemann solver. 
a)

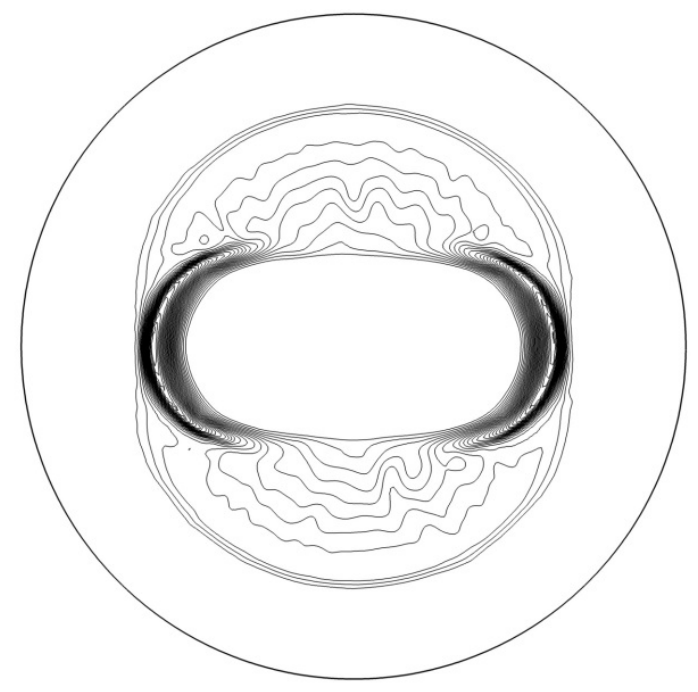

c)

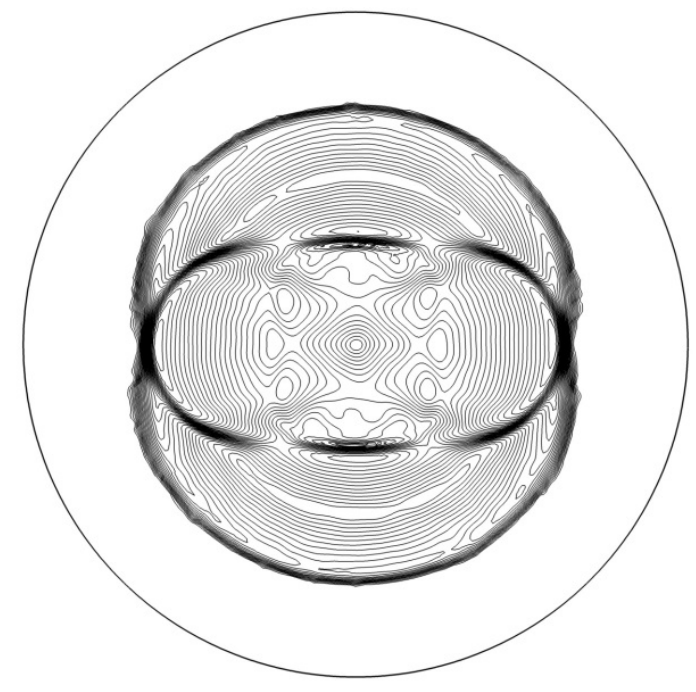

b)

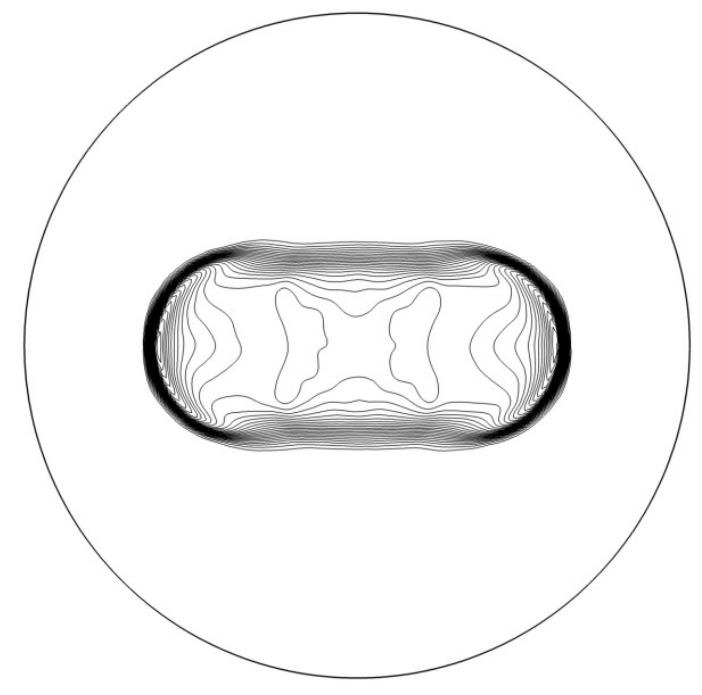

d)

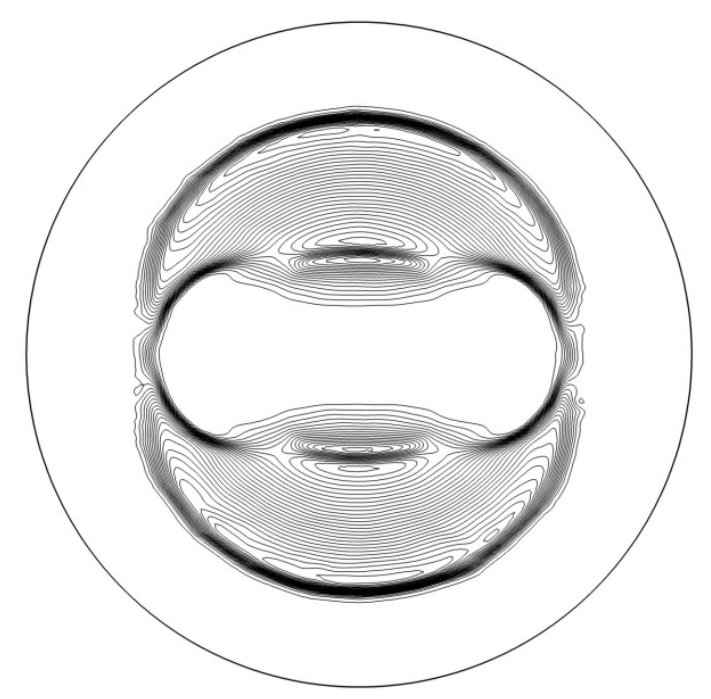

Figs. $7 a, 7 b, 7 c$ and $7 d$ show the density, pressure, velocity magnitude and magnetic field magnitude for the two-dimensional blast problem. A third order divergence-free ADER-WENO scheme was used along with the MuSIC Riemann solver . 
a)

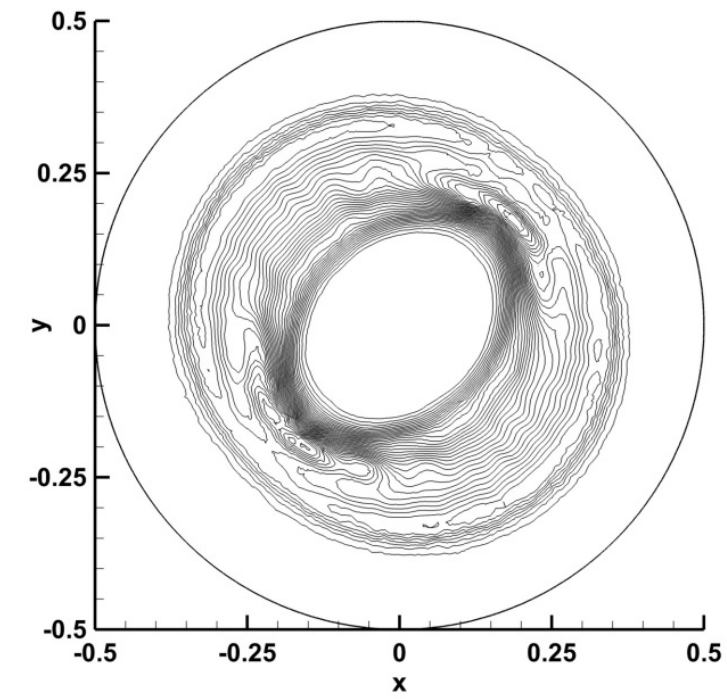

c)

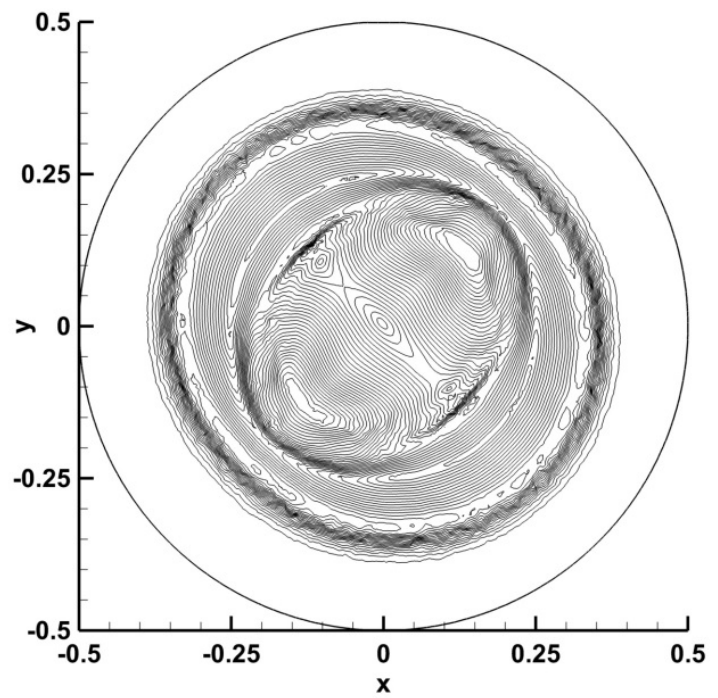

b)

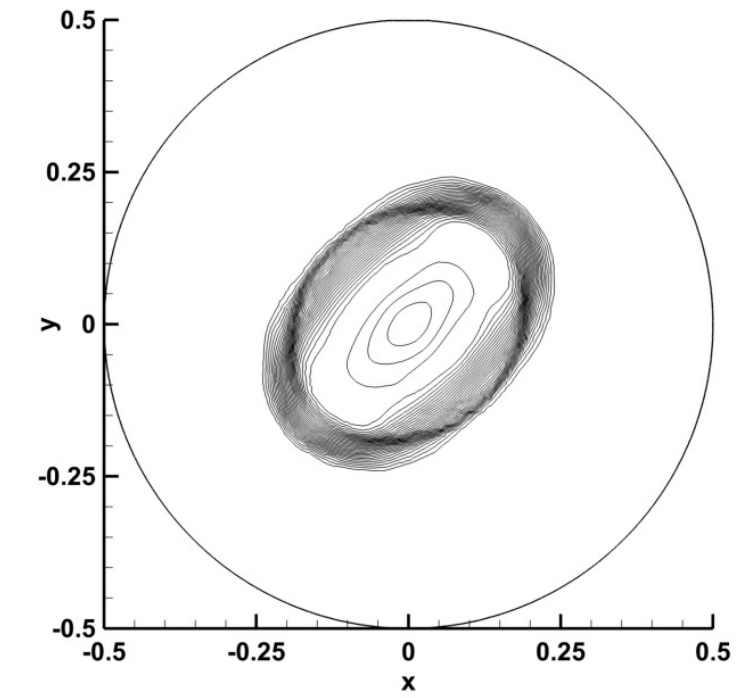

d)

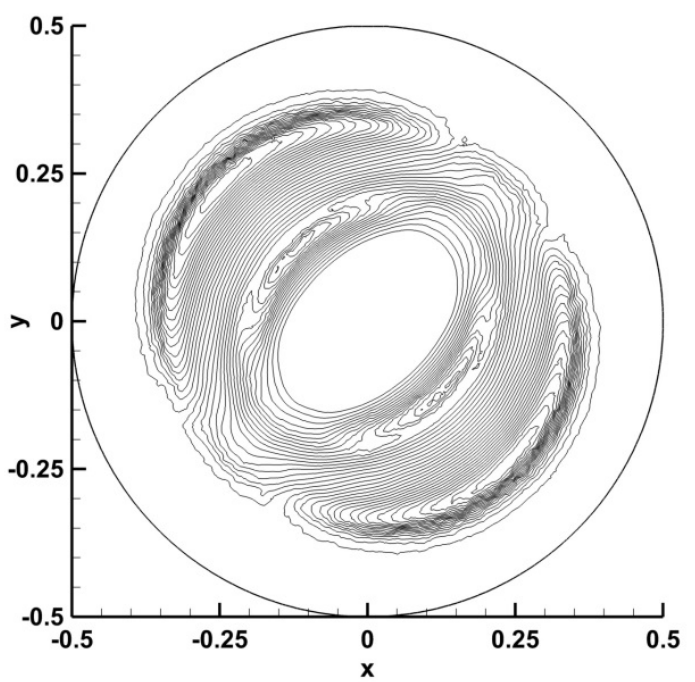

Figs. $8 a, 8 b, 8 c$ and $8 d$ show the density, pressure, velocity magnitude and magnetic field magnitude for the three-dimensional blast problem. A third order divergence-free ADER-WENO scheme was used along with the MuSIC Riemann solver . 


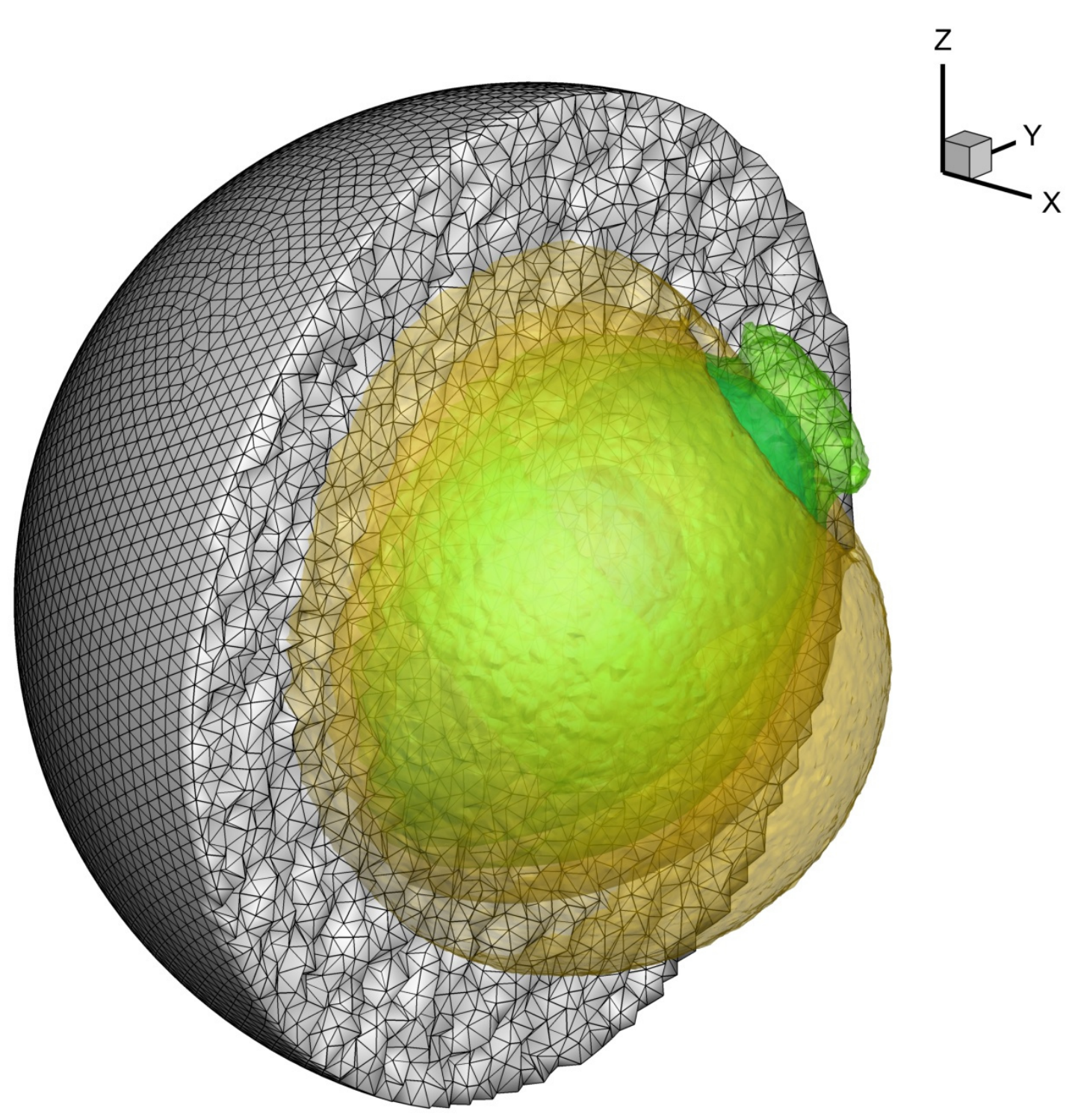

Fig. 9 shows a sketch of the unstructured tetrahedral mesh together with the isocontour surfaces of the magnetic field intensity for the three-dimensional blast problem. 

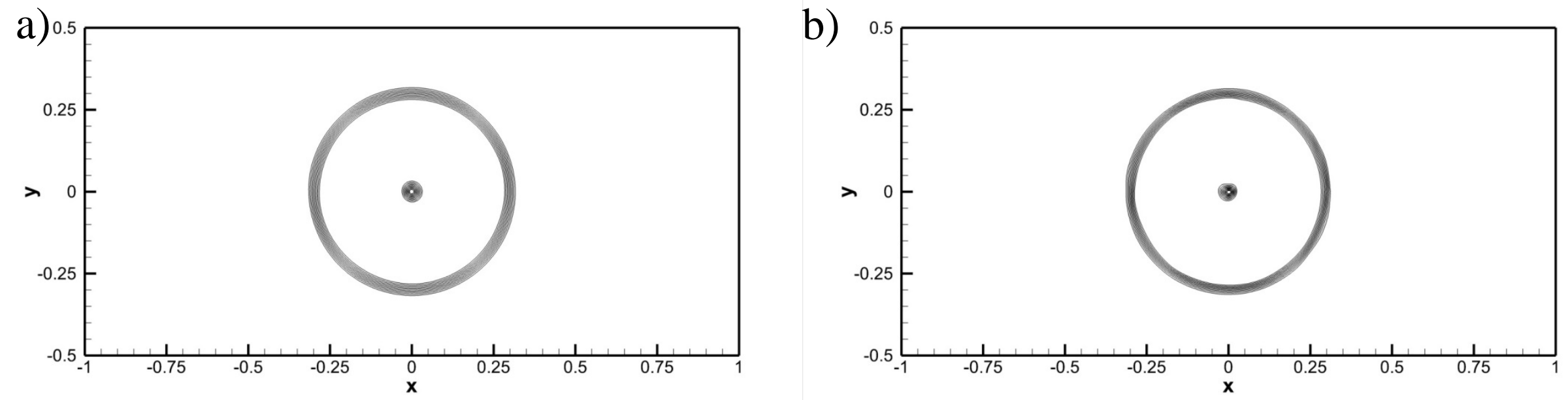

Fig. 10 shows the result of the field loop advection problem in two-dimensions. The resulting magnitudes of the magnetic field are shown in Fig. 10a and 10b for the third and fourth order cases respectively. The fourth order results are sharper than the third order results, as expected. 
